\title{
COLLISION-INDUCED LIGHT SCATTERING \\ IN LIQUIDS AND THE BINARY \\ COLLISION MODEL
}

\author{
A Thesis \\ Submitted to \\ the Faculty of Graduate Studies \\ University of Manitoba
}

In Partial Fulfillment

of the Requirements for the Degree

Master of Science

by

James Hon $\mathrm{Ki}$ Ho

August 1973

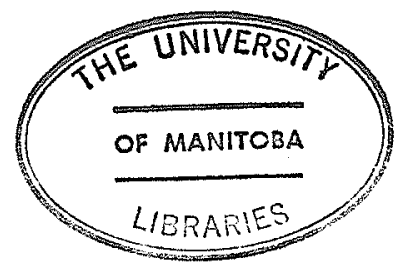


ACKNOWLEDGEMENTS

The author wishes to express sincere thanks to his research supervisor, Dr. G.C. Tabisz, for his friendly guidance throughout the whole period of this study.

Thanks are also extended to Dr. W.R. Wall for his advice concerning the computer programmes and $M r$. D.P. Shelton for his assistance in performing the experiments. 


\section{Abstract}

The collision-induced Rayleigh wing in the depolarized light scattering spectrum of five molecular liquids has been studied in order to try to assess the validity of the isolated binary collision model, currently used to explain the phenomenon.

Following the introduction to some fundamental principles of light scattering, models used to account for the induced anisotropic polarizability are discussed. The experimental methods are then described. The intensity profiles have been analyzed in terms of the expressions derived by Bucaro and Litovitz, $I(\omega) \propto \omega^{x} \exp \left(-\omega / \omega_{0}\right)$, and by Shin, $I(\omega) \propto \omega^{y} \exp \left(-C \omega^{12 / 19}+D \omega^{6 / 10}\right)$. It is concluded that before a positive distinction can be made between models or before reliable information on molecular dynamics may be extracted from the spectrum, a good theoretical estimate of the form of the induced polarizability $\Delta \alpha$ for close collisions is required. Further experimental studies of collision-induced scattering with isotropic gases at low densities are suggested. 
Acknowledgements . . . . . . . . . . . . . . . . i i

Abstract . . . . . . . . . . . . . . . . ii

Chapter 1 Fundamentals of Light Scattering . . . . . 1

1.1 Classical Intensity of Radiation from an Induced Dipole . . . . . . . . . 1

1.2 Average Over Al1 Orientations ...... 6

1.3 Classical Theory of the Raman Effect . . . . 8

1.4 Quantum Theory of the Raman Effect ..... 9

Chapter 2 Collision-Induced Light Scattering . . . . 12

2.1 Dipole-Induced Dipole Model . . . . . . 15

2.2 Electron Overlap Mode1......... I6 16

2.3 Frame Distortion Mode1......... 16

Chapter 3 Experimental Methods . . . . . . . . 19

3.1 The Source and I1lumination System . . . . 19

3.2 The Spectral Analyzer or Monochromator . . . 23

3.3 Radiation Detection .......... 24

3.4 Frequency Calibration of the Spectrometer. . 25

Chapter 4 Method of Analysis ........... 28

4.1 Method of Least Squares ......... 28

4.2 The Gauss Method of Solving the Nonlinear Problem ............... 44

4.3 Details of the Computer Programme . . . . 46 
Chapter 5 Analysis and Discussion . . . . . . . 49

5.1 Bucaro and Litovitz Formulation for the Intensity of the Scattered Radiation . . . 49

5.2 Shin Formulation of the Scattered Intensity . 54 Chapter 6 Summary .............. . 64

References . . . . . . . . . . . . . . 69

Appendix 1 The Computer Programme . . . . . . . . 71

Appendix 2 Publication Reprint.......... 78 
CHAPTER 1

FUINDAMENTALS OF LIGHT SCATTERING

When light passes through a medium, photons are scattered in all directions. The scattered radiation originates in oscillating electric dipole moments induced by the incident light field, which are further modulated by processes intrinsic to the medium. Consequently, the scattered light contains information on the medium.

\subsection{Classical Intensity of Radiation from an Induced Dipole}

An induced dipole can emit radiation just as a permanent oscillating dipole. If the molecules in the sample are at rest, the scattered light has the same frequency as the incident 1ight; this is Rayleigh scattering. However, if the molecule, for example, is vibrating, the scattered radiation, in addition to the incident frequency, consists of frequencies equal to the sum and to the difference of the incident frequency and the frequency of the internal motion; this is Raman scattering. If $\omega$ is the angular frequency of oscillation of the emitted radiation, c the velocity of light, and $\mu_{0}$ the amplitude of the induced dipole $\mu$, where

$$
\mu=\mu_{0} \cos \omega t,
$$

then the mean rate of total radiation is given by (Wilson et al.1955)

$$
I=\frac{\omega^{4}}{3 c^{3}} \mu_{0}^{2}
$$

If one introduces nonrotating axes, which are parallel to the spacefixed axis of the observer but whose origin translates with the 
molecular center of mass, the total radiation emitted per unit solid angle in the $X$ direction is (Wilson et al. 1955).

$$
I=\frac{\omega^{4}}{8 \pi c^{3}}\left(\mu_{o y}^{2}+\mu_{o z}^{2}\right)
$$

If the electric field strength is $\vec{E}$ and the induced moment is $\vec{\mu}$, then

$$
\vec{\mu}=\alpha \vec{E}
$$

where $\alpha$ is the polarizability for an isotropic molecule. However, if the molecule is anisotropic, equation (1.3) must be replaced by the more complex expressions

$$
\begin{aligned}
& \mu_{x}=\alpha_{x x} E_{x}+\alpha_{x y} E_{y}+\alpha_{x z} E_{z}, \\
& \mu_{y}=\alpha_{y x} E_{x}+\alpha_{y y} E_{y}+\alpha_{y z} E_{z}, \\
& \mu_{z}=\alpha_{z x} E_{x}+\alpha_{z y} E_{y}+\alpha_{z z} E_{z} .
\end{aligned}
$$

The quantities $\alpha_{i j}$ are dependent upon the orientation of the molecule relative to the nonrotating axes and form the components of the polarizability tensor. They are properties of the molecule and are independent of the components of the electric vector.

From equation (1.2) the intensity, I, of the scattered light can be expressed in terms of the components of the polarizability tensor by substitution of the appropriate expressions for $\mu_{\text {oy }}^{2}$ etc. Assuming the incident light propagates along the $Y$-axis and with its electric vector vibrating along the $x$-axis which is also the direction of observation, then the following expression can be obtained by using 
equations (1.2) and (1.4),

$$
I_{T}(\text { obs. } \|)=\frac{\omega^{4}}{8 \pi c^{3}}\left(\alpha_{y x}^{2}+\alpha_{z x}^{2}\right) E_{o}^{2}
$$

where $\mathrm{I}_{\mathrm{T}}$ (obs. $\|$ ) is the scattered intensity when the incident light is polarized in the X-direction. Similarly the scattered intensity for the incident light polarized in the $Z$-direction, $\left(I_{T}\right.$ obs. $\left.\perp\right)$, is (Fig. 1)

$$
I_{T}(\text { obs. } \perp)=\frac{\omega^{4}}{8 \pi c^{3}}\left(\alpha_{y z}^{2}+\alpha_{z z}^{2}\right) E_{o}^{2} .
$$

From equation (1.6) the component of the scattered light which is polarized parallel to $\mathrm{E}$ is, if the electric vector is in the $\mathrm{Z}$ direction,

$$
\left.\left.I \|^{(o b s} \cdot\right\rfloor\right)=\frac{\omega^{4}}{8 \pi c^{3}} \alpha_{z z}^{2} E_{o}^{2}
$$

Expressing equations (1.5), (1.6) and (1.7) in terms of the incident intensity $I_{0}$ whose electric vector has amplitude $E_{0}$, the following equations can be obtained,

$$
\begin{aligned}
& I_{T}(\text { obs. } \|)=\frac{\omega^{4}}{c} I_{0}\left(\alpha_{y x}^{2}+\alpha_{z x}^{2}\right), \\
& I_{T}(o b s . \perp)=\frac{\omega^{4}}{c^{4}} I_{0}\left(\alpha_{y z}^{2}+\alpha_{z z}^{2}\right), \\
& I_{H}(\text { obs. } \perp)=\frac{\omega^{4}}{c^{4}} I_{0} \alpha_{z z}^{2}
\end{aligned}
$$

since $I_{0}$, from electromagnetic theory, is given by

$$
I_{0}=\frac{c}{8 \pi} \quad E_{0}^{2}
$$




\section{Figure 1}

Schematic representation of experimental conditions defining $I_{T}$ (obs. $\|$ ), $I_{T}$ (obs. $\perp$ ), and $I_{\|}$(obs. $\perp$ ) 

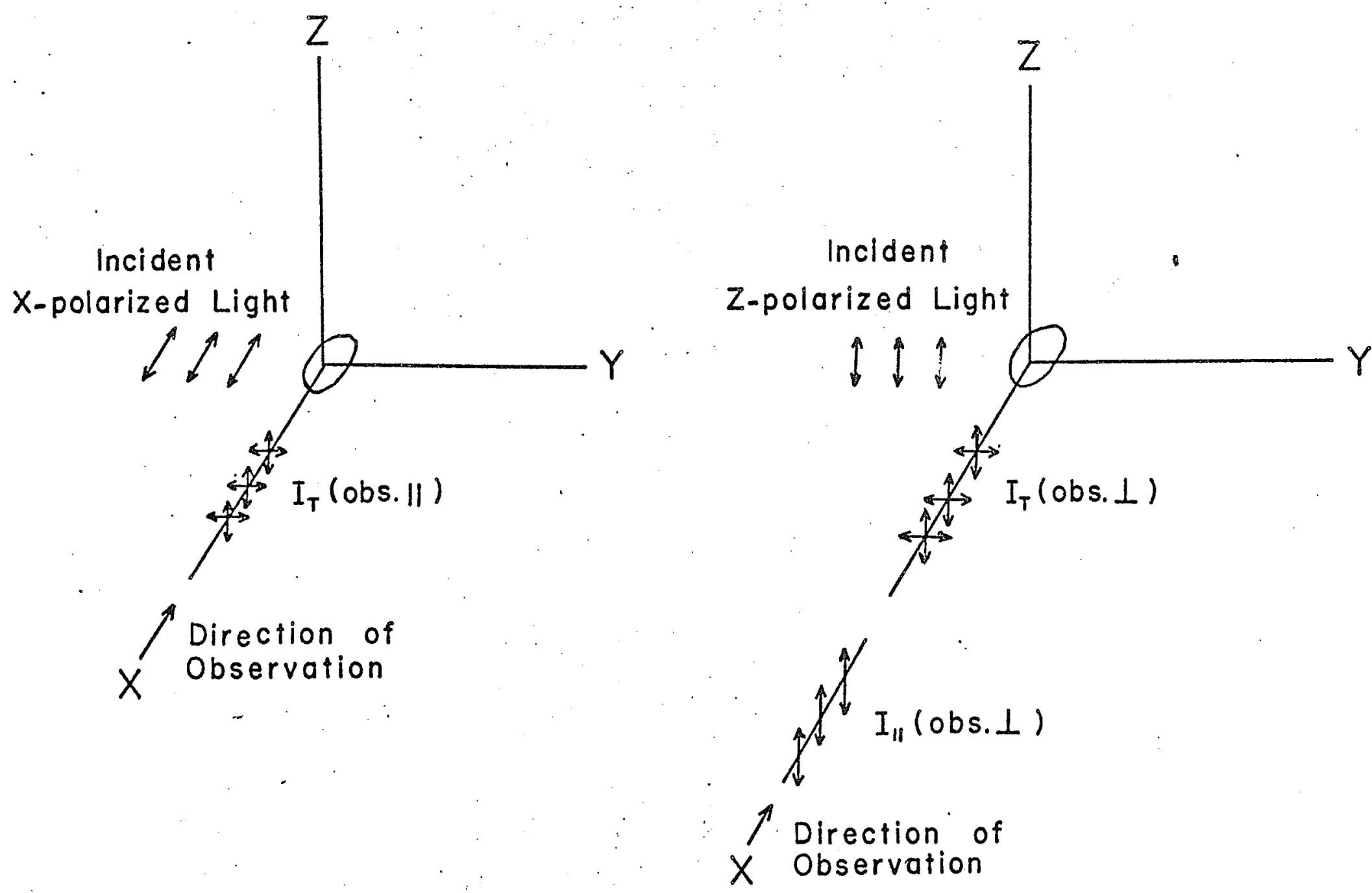
If the molecule is isotropic, equation (1.8) will give $I_{T}$ (obs. $\left.\|\right)=0$ because all the elements of the polarizability tensor are zero except on the diagonal. This applies only to free molecules. In dense media in which the isotropy is destroyed by interactions, this component is not necessarily zero. Such a non-zero component of scattered light forms the basis of collision induced light scattering.

\subsection{Average Over All Orientations}

Equations (1.8), (1.9) and (1.10) are only valid for a single molecule. To account for an ensemble of molecules with all possible orientations with respect to the observer's fixed axes, these equations have to be multiplied by the number of molecules and the terms $\alpha_{i j}^{2}$ averaged over all orientations of the principal axes with respect to the fixed $X, Y, Z$ axes. The principal axes are the axes in the molecule such that the relation between $\mu$ and $E$, when referred to them, assumes the simple form:

$$
\begin{aligned}
& \mu_{1}=\alpha_{1} E_{1}, \\
& \mu_{2}=\alpha_{2} E_{2}, \\
& \mu_{3}=\alpha_{3} E_{3} .
\end{aligned}
$$

Equations (1.8), (1.9) and (1.10) will then be changed to

$$
\begin{aligned}
& I_{T} \text { (obs.|.|) }=\frac{1}{15} \frac{\omega^{4}}{c^{4}} N I_{0}\left(2 \sum_{i} \alpha_{i}^{2}-2 \sum_{i<j} \alpha_{i} \alpha_{j}\right), \\
& I_{T}(\text { obs. } \mid .)=\frac{1}{15} \frac{\omega^{4}}{c^{4}} N I_{0}\left(4 \sum_{i} \alpha_{i}^{2}+\sum_{i<j} \alpha_{i} \alpha_{j}\right),
\end{aligned}
$$




$$
\left.I \|^{(o b s} \cdot 1\right)=\frac{1}{15} \frac{\omega^{4}}{c^{4}} N I_{0}\left(3 . \sum_{i} \alpha_{i}^{2}+2 \sum_{i<j} \alpha_{i} \alpha_{j}\right)
$$

The spherical part of the polarizability, $\alpha_{0}$, and the anisotropy, $\beta$, are defined by

$$
\begin{aligned}
& \alpha_{0}=\frac{1}{3}\left(\alpha_{1}+\alpha_{2}+\alpha_{3}\right) \\
& \beta^{2}=\frac{1}{2}\left[\left(\alpha_{1}-\alpha_{2}\right)^{2}+\left(\alpha_{2}-\alpha_{3}\right)^{2}+\left(\alpha_{3}-\alpha_{1}\right)^{2}\right] .
\end{aligned}
$$

If the summation in equations (1.11), (1.12) and (1.13) are replaced by the expressions (1.14) and (1.15), then

$$
\begin{aligned}
& I_{T}(\text { obs. } \|)=\frac{\omega^{4}}{c^{4}} N I_{0} \frac{2 \beta^{2}}{15}, \\
& \left.I_{T} \text { (obs. } \perp\right)=\frac{\omega^{4}}{c^{4}} N I_{0} \frac{\left(45 \alpha_{o}^{2}+7 \beta^{2}\right)}{45}, \\
& \left.I_{\|} \text {(obs. } \perp\right)=\frac{\omega^{4}}{c^{4}} N I_{0}\left(\frac{45 \alpha_{o}^{2}+4 \beta^{2}}{45}\right)
\end{aligned}
$$

The depolarization ratios $\rho_{1}$ and $\rho_{n}$ are defined as the ratio of the scattered intensity which is polarized perpendicular to $E$ to the intensity parallel to $E$. With linearly polarized incident light and observations made in a direction perpendicular to $E \quad \rho_{1}$ is given by

$$
\rho_{1}=\frac{I_{T}(\text { obs } \cdot 1)-I \mid(\text { obs } \cdot 1)}{I_{1}(\text { obs } \cdot 1)}=\frac{3 \beta^{2}}{45 \alpha_{0}^{2}+4 \beta^{2}}
$$

Hence for free isotropic molecules $\rho_{1}=0$. For natural (unpolarized) light $\rho_{\mathrm{n}}$ is 


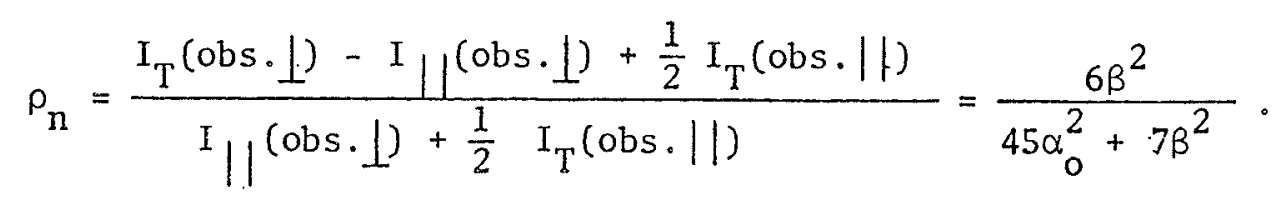

\subsection{Classical Theory of the Raman Effect}

The Raman effect is due to inelastic scattering from gases, liquids or solids. Again, if an atom or molecule is brought into an oscillating electric field $\mathrm{E}$ of the incident light,

$$
E=E_{0} \sin \omega^{\prime} t
$$

where $E_{0}$ and $\omega^{s}$ are the amplitude and the angular frequency of the electric field respectively and $t$ is the time, a varying dipole moment $\vec{\mu}$ will be induced such that

$$
\vec{\mu}=\alpha \overrightarrow{\mathrm{E}}
$$

This varying dipole moment will in turn emit light of the same frequency as the incident light giving Rayleigh scattering.

Vibrational motion would change the internuclear distance. This change would in turn modulate the polarizability and thus modulate the amplitude of the induced dipole moment. Hence $\alpha$ can be written as

$$
\alpha=\alpha_{\text {ov }}+\alpha_{1 v} \sin \omega_{\text {osc }} .^{\imath}
$$

where $\alpha_{\text {ov }}$ is the polarizability in the equilibrium position and $\alpha_{1 v}$ is the amplitude of the change in polarizability during the vibration. Substituting equations (1.23) and (1.21) into (1.22), the following 
expression is obtained for the induced dipole moment in the case of a vibrating molecule,

$$
\mu_{v}=\alpha_{o v} E_{o} \sin \omega^{\prime} t+\alpha_{1 v_{o}} E_{0} \sin \omega^{\prime} t \sin \omega_{o s c} t
$$

Rearranging the above expression one has

$$
\mu_{v}=\alpha_{o v} E_{o} \sin \omega^{\prime} t+\frac{1}{2} \alpha_{1 v} E_{o}\left[\cos \left(\omega^{\prime}-\omega_{o s c}\right) t-\cos \left(\omega^{\prime}+\omega_{o s c}\right) t\right]
$$

Hence the induced dipole moment oscillates with the angular frequencies $\omega^{r}$ s $\omega^{r}-\omega_{\text {osc }}$ and $\omega^{\prime}+\omega_{\text {osc }}$ on account of the small change of $\alpha$ during the vibration. So there will be displaced lines on both sides of the Rayleigh line in the total spectrum. When the same procedure is applied to the rotational motion it may be shown that a rotational spectrum with displaced lines at frequencies $\omega^{\prime}-2 \omega_{\text {rot }}$ and $\omega^{\prime}+2 \omega_{\text {rot }}$ is obtained in addition to the Rayleigh line. The lines which are displaced toward lower frequencies are called Stokes lines while those displaced toward higher frequencies are called anti-Stokes lines. Fig. 2 is a spectrum of the liquid carbon tetracholoride and shows clearly the Rayleigh line and two Stokes vibrational lines. The one that is displaced to higher frequencies from the Rayleigh line is due to the $v_{4}$ vibration while the other one is due to the $v_{2}$ vibration.

\subsection{Quantum Theory of the Raman Effect}

When a photon of energy $\hbar \omega$ interacts with an atom or molecule, it can be scattered either elastically or inelastically. If it is scattered elastically there would be no change in the energy of the 
photon. This gives the Rayleigh effect. For inelastic scattering the photon can either absorb some energy from the atom or give up some of its energy to the atom. In the first case the energy of the scattered photon is $\hbar \omega^{\prime}+\Delta E$ where $\Delta E=E^{\prime}-E^{\prime \prime}$ and the atom which was initially in the higher state $E^{\prime}$ will go to the lower state E", while in the second case the energy of the: scattered photon is $\hbar \omega^{\prime}-\Delta \mathrm{E}$ and the atom is brought to the higher state $E^{\prime}$ from the lower state E" by the scattering. The frequencies of the scattered light quantum are given by $\omega^{8}+\frac{\Delta E}{\hbar}$ and $\omega^{8}-\frac{\Delta E}{\hbar}$ (Raman effect). Classically, the intensities of the Stokes lines $\left(\omega^{\prime}-\omega_{\text {osc }}\right)$ to the anti-Stokes lines $\left(\omega_{0}+\omega_{o s c}\right)$ should be simply the ratio

$$
\frac{I_{\text {Stokes }}}{I_{\text {anti-Stokes }}}=\frac{\left(\omega^{\prime}-\omega_{\text {osc }}\right)^{4}}{\left(\omega^{\prime}+\omega_{\text {osc }}\right)^{4}}
$$

This is in contradiction to the experimental findings which show that the Stokes lines are more intense than the anti-Stokes lines. Quantum mechanically the ratio of the intensities is correctly given by

$$
\frac{I_{\text {Stokes }}}{I_{\text {anti-Stokes }}}=\left(\frac{\omega^{\prime}-\omega_{n^{\prime \prime} n^{\prime}}}{\omega^{\prime}+\omega_{n^{\prime \prime} n^{\prime}}}\right)^{4} \frac{N_{n^{\prime \prime}}}{N_{n^{\prime}}} \frac{g_{n^{\prime}}}{g_{n^{\prime \prime}}}
$$

where $n^{\prime}$ is the upper state and $n^{\prime \prime}$ is the lower state, $N_{n^{\prime \prime}}$ is the number of molecules in the state described by the quantum number $\mathrm{n}^{\prime \prime}$ and $\mathrm{g}_{\mathrm{n}^{\prime \prime}}$ is the degeneracy. The population ration is given by the Boltzmann factor

$$
\frac{N_{n^{\prime \prime}}}{N_{n^{\prime}}} \frac{g_{n^{\prime}}}{g_{n^{\prime \prime}}}=e^{\left(W_{n^{\prime}}-W_{n^{\prime \prime}}\right) / k T}
$$

with $W_{n^{\prime}}>W_{n^{\prime \prime}}$ where $W_{n^{\prime}}$ and $W_{n^{\prime \prime}}$ are the energies of the upper 
and lower states respectively. Since the lower state is more densely populated than the upper state, the Stokes lines are more intense than the anti-Stokes Iines.

Just as changes in the internal vibrational and rotational energy of the molecule lead to vibrational and rotational Raman effects, the relative kinetic energy of a system of colliding molecules may be altered in a scattering process giving rise to a translational Raman effect. Collision-induced scattering, the subject of this thesis, is such a translational Raman effect and is described in the following chapter. 
CHAP'TER 2

\section{COLLISION-INDUCED LIGHT SCATTERING}

Collision-induced light scattering manifests itself as a depolarized component to the Rayleigh wing in the light scattering spectrum. Fig. 2 is a typical spectrum of liquid carbon. tetrachloride which shows clearly this broad wing on the Rayleigh line.

It 1. ; been shown in equations (1.19) and (1.20) that for free, isotropic molecules the depolarization ratio is zero and therefore light Rayleigh scattered from free, optically isotropic atoms or molecules is polarized. However, when atoms interact with one another, an induced anisotropy in the total polarizability of the system can arise. Recent experiments (Levine et al. 1968; Thibeau et al. 1968; McTague et a1. 1969; Gornall et al. 1970) have shown that a broad, depolarized wing occurs on the Rayleigh line in liquids or dense gases. A quadratic density dependenco of the intensity at moderate gas pressures and an exponential decrease in the intensity with increasing frequency for a number of rare"gases and simple liquids was also reported. For the far wing the depolarization ratio $\rho_{1}$ is approximately $3 / 4$ implying an induced anisotropy.

Theoretical studies on collision-induced light scattering spectra have been carried out (Buckingham et a1. 1957; Levine et al. 1968; Thibeau et al. 1968). This phenomenon has been ascribed to an incremental polarizability $\Delta \alpha$ induced during molecular collisions. It is often assumed in the binary model that $\Delta \alpha$ has a $r$-dependence of the form

$$
\Delta \alpha \propto r^{-m}
$$


13.

\section{Figure 2}

Light scattering spectrum of liquid carbon tetrachloride showing:-
(A) the Rayleigh line,
(B) the broad depolarized wing,
(C) the Stokes lines. 


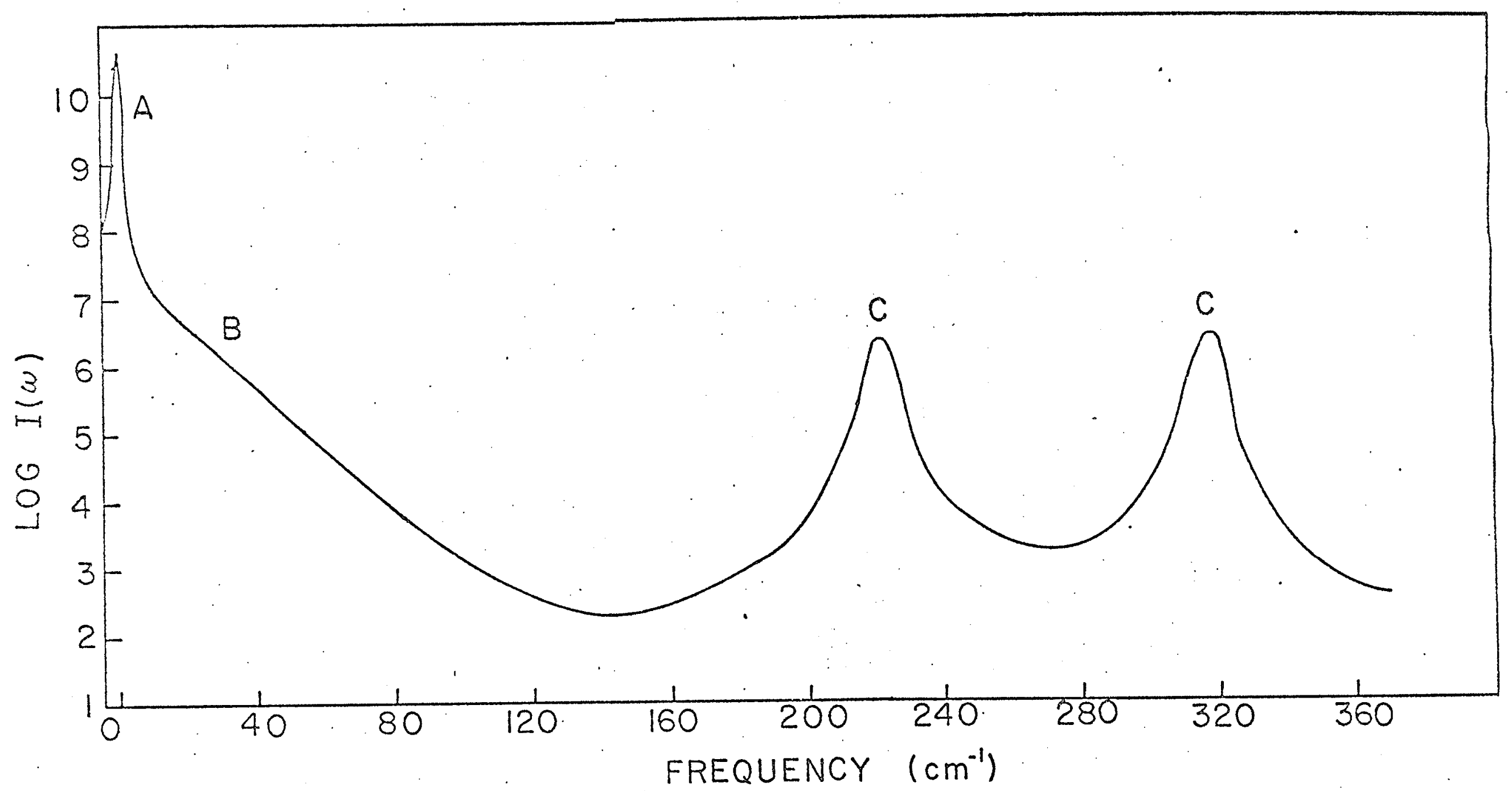

$=$ 
where $r$ is the distance between the atoms or molecules and $m$ is some integer. Three models have been used to account for the anisotropic polarizability.

2.1. Dipole-Induced Dipole Mode1 (Buckingham et al. 1957; Thibeau et a1. 1968; Gersten 1971) (DID)

In this model the interaction between the induced electric dipoles causes the anisotropy. An induced dipole moment is created on an atom by the incident field. A neighbouring atom also having an induced moment, and being in the field of the first dipole, induces an additional small moment. Starting with the expression for the field of an electric dipole, Thibeau et al. (1968) and Lallemand (1970) derived an expression for the dipole induced by a neighbouring dipole. Having worked out the components of the polarizability tensoi using the near field approximation, they arrived at the result that the induced anisotropy $\Delta \alpha$ is given by

$$
\Delta \alpha=\frac{6 \alpha^{2}}{r^{3}}
$$

where $r$ is the interatomic distance. The $\alpha$ in equation (2.1) is a property of the individual molecule while the induced anisotropy, $\Delta \alpha$, is a property of the system of interacting molecules. To account for the scattering $\alpha$ is replaced by $\Delta \alpha(r)$ in equations (1.16) to (1.20). Note that in this model $\Delta \alpha(x)$ is completely anisotropic, that is $\alpha_{0}(r)=0, \quad \beta(r)=6 \alpha^{2} / r^{3}$. Hence this is a long-range interaction; the polarizability varies as the inverse cube of the interaction distance. The polarizability distortion for moderate separations of the two atoms can be explained by this model. Gersten (1971) worked 
out a theory for the asymptotic line shape in collision-induced light scattering in argon and krypton and showed that the wings of the spectrum cannot be fitted by a single exponential curve. It was found that when the intensity was plotted semilogarithmically there was a steep slope at low frequencies and a less steep slope at higher frequencies. Gersten's theoretical prediction was verified experimentally by Slusher, Surko and Strautins.

\subsection{Electron Overlap Model (Levine et a1. 1968; McTague et al. 1971)(EO)} When two atoms collide, they deform because of overlap of electron clouds and their polarizability is no longer spherically symmetric causing depolarized scattering. Such a distortion is also responsible for an induced polarizability. Originally, the variation of the incremental polarizability with interatomic separation $r$ was assumed to be

$$
\Delta \alpha \propto r^{2} \exp \left(-a r^{2}\right)
$$

by Levine and Birnbaum (1968). This expression exhibits the proper asymptotic behaviour, $\Delta \alpha(r) \rightarrow 0$ as $r \rightarrow \infty$ and $\Delta \alpha(r) \rightarrow 0$ as $r \rightarrow 0$. Recently the induced anisotropy was calculated to have a $r^{-9}$ dependence in the case of argon by Vukcevich (1971) using a deformable shell model. This effect is related to the repulsive forces between two molecules and hence is a strong function of $r$.

\subsection{Frame Distortion Model (Gabelnick et a1. 1968)}

Frame distortion is the distortion of molecular structure by a change in the bond length or the bond angle or both. With 
the assumption that it is proportional to short-range force and using a Lennard-Jones potential, molecular frame distortion during close collisions gives a pair polarizability varying as $\mathrm{r}^{-13}$

Litovitz et al. (1971) assert that a cancellation of the DID effects occurs at liquid densities and that the EO effect is essentially a binary process because even in a multiple collision a molecule has a low probability of strongoverlap with more than one neighbor. Hence the EO effect will be additive in a pairwise sense. Bucaro and Litovitz (1971), the first to apply low density. models to liquids, met with considerable success. They assumed that the angular dependence of the induced polarizability could be neglected; that is, $\Delta \alpha$ is simply proportional to $\mathrm{r}^{-\mathrm{m}}$. In addition, they considered the interactions effective in producing the scattering to be of short range i.e. $m>1$. The neglect of long-range contributions to $\Delta \alpha$ may be good assumption for highly dense media as there is experimental evidence (Thibean et a1. 1968) that the scattered intensity due to the DID mechanism decreases as the medium becomes ordered. With these assumptions, Bucaro and Litovitz were able to arrive at a compact expression to describe the dependence of intensity, $I$, on frequency, $\omega$, in the wing of the profile of the scattered light,

$$
I(\omega) \propto \omega^{2(m-7) / 7} \exp (-\omega / \omega 0)
$$

Following their efforts, Shin (1972) has developed a more exact analytical expression for the intensity within this so-called isolated binary collision (IBC) model, 


$$
I \propto \omega^{(2(m-7) / 7)+(4 / 19)} \exp \left(-C \omega^{12 / 19}+D \omega^{6 / 19}\right)
$$

Both equations (2.4) and (2.5) are discussed further in chapter 5. Preliminary reports (Tabisz et al. 1972) have shown that there are difficulties in the interpretation when applying these calculated line shapes to liquid spectra. As well, there have been recent suggestions that the isolated binary collision model is not appropriate in dense media and higher order interactions must be included (Pasmanter et al. 1972; Gelbart 1972). Therefore, a study of the profiles of the collision-induced scattering from several molecular liquids has been carried out in order to assess the binary collision model. 
CHAPTER 3

EXPERIMENTAL METHODS

The experimental set-up is typical of laser Raman scattering studies and can be conveniently separated into three systems:

(1) the source and illumination system,

(2) the spectral analyser or monochromator, and

(3) the radiation detection system.

Light from an argon-ion laser is passed through a half-wave plate and prism polarizer and focussed in the sample cell. The light scattered at $90^{\circ}$ is collected and brought to a scanning double monochromator before reaching the detector. The output of the detector is stored in a multichannel analyser. A block diagram of the experimental setup is presented in Fig. 3 .

\subsection{The Source and Illumination System}

An argon-ion laser (CRL \#52) operating at about $3 / 4$ watt at $4880^{\circ}$ is used as the exciting illumination. The laser beam is passed through a half-wave plate and prism polarizer before being focussed into the sample cell. By focussing the laser beam, high power densities can be obtained. Since the intensity of the scattered radiation depends on the power densities, the increased radiance obtained by focussing the laser beam will offset the disadvantage of having a small sample.

The laser has the following advantages as a source of exciting radiation:

(1) The intensity of the laser beam is high.

(2) The polarization of the laser beam is well-defined. 


\section{Figure 3}

A block diagram of the experimental set-up. 


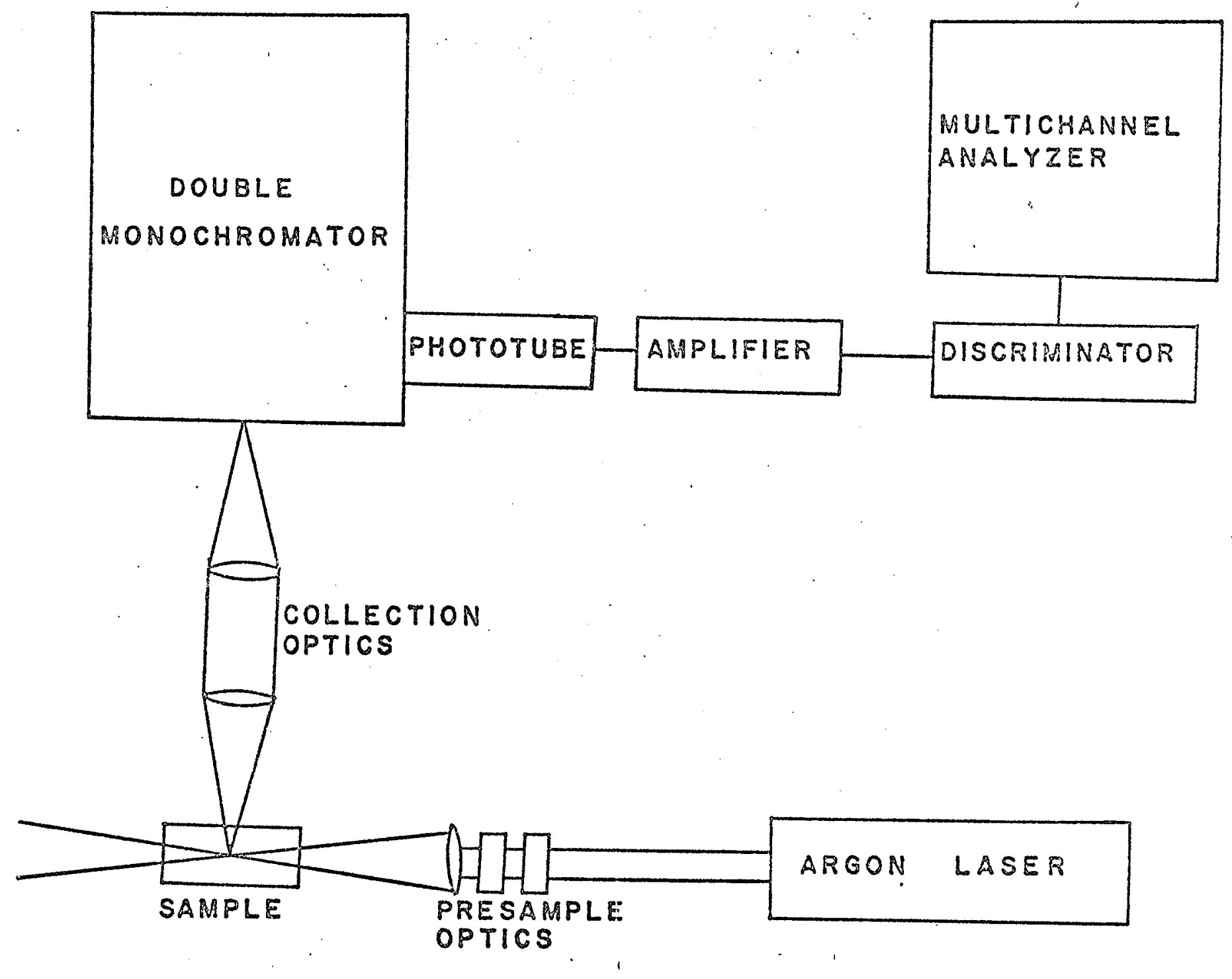


(3) A particular laser line is very sharp in frequency and several exciting lines are available. For the argon ion laser the radiations normally used are the $4880 \AA$ and $5145 \AA$. The $4880 \AA$ A line was chosen here because the scattered intensity is proportional to $\omega^{4}$ and the sensitivity of the detector is higher than at $5145 \stackrel{\circ}{\mathrm{A}}$.

(4) The laser produces its radiation in a well collimated beam of very small cross-section. As a result the flux density in a small sample can be extremely high. The illumination geometry can be designed to take advantage of this concentration.

The geometry used is such that if the incident light is considered to travel in the $\mathrm{Y}$-direction, polarized in the $\mathrm{X}$-direction, then observations are made in the X-direction. This geometry is used because the Rayleigh line, which is caused predominately by isotropic scattering, can be decreased if the scattered radiation is observed in a direction that coincides with the direction of vibration of the electric vector of the exciting radiation. Here use is made of the well-defined polarization of the laser beam. Hence the scattered radiation is observed at 90 degrees to the laser beam and the beam is polarized parallel to the observation direction. The half-wave plate is used to turn the direction of polarization by $90^{\circ}$ and the prism polarizer ensures that all the light going into the sample cell is linearly polarized and also corrects for the depolarization caused by the half-wave plate. The degree of depolarization of the incident beam is now less than $1 \%$. The liquid sanple is passed 
through a membrane filter of diameter 0.2 microns before being introduced into the fused quartz cell of dimensions $1 \times 1 \times 5 \mathrm{~cm}$. Since fused quartz has no definite crystal structure, the light passing through the walls of this cell will not be depolarized. This filtering process is necessary to remove the dust particles which may cause Tyndall scattering.

\subsection{The Spectral Analyzer or Monochromator}

The scattered radiation coming out from the sample cell is collected by a $f / 2$ system and then brought into a double scanning monochromator (Jarrel1-Ash \#25-100) which is composed of an entrance slit, a system of gratings and an exit slit. The light after passing through the first lens is parallel and is then focussed by the second lens onto the entrance slit of the monochromator in such a way that the entrance cone matches with the acceptance cone of the monochromator. Since Raman radiations are weak relative to the radiation at the exciting frequency, Raman spectrometers must be free of stray light which may arise from a few sources such as leakage of light from the instrument housing, leakage of light through the slits which does not come from the sample, etc. The double monochromator, which is one monochromator followed by and coupled to a second, is used in the experiment because it has the advantage of reducing the stray exciting radiation over the single monochromator. Even in a carefully designed single monochromator there is always a large amount of stray light present inside the monochromator arising from the reflection off the gratings. The unwanted radiation which escapes through the exit slit of the first monochromator along with the Raman radiation is redispersed 
and rejected in the second part of the double monochromator. After passing through the system the amount of stray lisht is reduced to $10^{-10}$ of the original amount at $42 \mathrm{~cm}^{-1}$ from the exciting frequency (from JACO manua1). However, our data was found to be sufficiently free of stray light and suitable for fitting at $6 \mathrm{~cm}^{-1}$ from the exciting frequency. The major, stray light is at the laser frequency.

A slit width of $1 \mathrm{~cm}^{-1}$ has been used. The stepping drive for the monochromator gratings controls the channel advance of the multichannel analyzer. This drive is linear in wavenumber and is so designed that 20 steps of the grating produce a change of $1 \mathrm{~cm}^{-1}$ in the frequency of the light appearing at the exit slit. The pulses generated in the drive circuit to perform the grating step are counted and after a predetermined number of steps have occurred, the multichannel analyzer is advanced by one channe1. In the present experiments, the monochromator is driven at a speed of $1 \mathrm{~cm}^{-1} / \mathrm{sec}$ and the channel of the multichannel analyzer is advanced every 10 steps. Each sample is scanned through a range of 200 wavenumbers and the scan repeated 10 times so that the counts from each scan are summed up for the 10 scans. This method of repetitive scanning is used to average out any change in physical conditions during the process of the experiment especially the fluctuation in laser intensity.

\subsection{Radiation Detection}

The Raman scattered radiation is then directed to a photosensitive detector (EMI 625613) which is usually composed of a photomultiplier with a photomissive cathode. The photomultiplier tube is cooled $\left(-20^{\circ} \mathrm{C}\right)$ and used in the photon-counting mode. Ilectron pulses, which are 
correlated to the photons striking the photocathode, occuring at the anode of the photomultiplier tube are individually amplified and counted. The photon counting mode is preferred to the other counting schemes because Raman signals are at a low level light intensity and the photon counting detection is the most sensitive (Young 1969). Photon counting detection yields a higher signal to noise ratio than the other counting schemes. The output from the amplifier includes both the photoelectron pulses and the noise pulses. The noise pulses are then reduced by the pulse height analyzer with the discriminator levels set for an optimum signal-to-noise ratio at low light intensities. The optimum signal-to-noise ratio was determined according to the method given by Young (1969). The output is stored in the multichannel analyzex (Victoreen \#ST400M) and the data can be printed out. The photomultiplier tube is cooled to reduce thermal emission from the photocathode.

\subsection{Frequency Calibration of the Spectrometer}

To calibrate the spectrometer a neon lamp with lines of known frequency is used. The spectrometer is then scanned over a range in which observations are also made. The wavenumber and the corresponding channel number are marked down when a line occurs. This process is then repeated for a number of different lines of neon. Hence the dispersion can be calculated by dividing the wavenumber difference between two lines by the corresponding channel difference. Avalue of

$0.496 \mathrm{~cm}^{-1} / \mathrm{ch}$ is obtained for the dispersion under experimental conditions. No non-1inearities in the drive were noted in the region of interest 
$\left(20500 \mathrm{~cm}^{-1}\right.$ to $\left.20250 \mathrm{~cm}^{-1}\right)$.

The liquids used in the experiment are chloroform $\left(\mathrm{CHCl}_{3}\right)$, benzene $\left(\mathrm{C}_{6} \mathrm{H}_{6}\right)$, toluene $\left(\mathrm{C}_{7} \mathrm{H}_{8}\right)$, cyclohexane $\left(\mathrm{C}_{6} \mathrm{H}_{12}\right)$ and carbon tetrachloride $\left(\mathrm{CCl}_{4}\right)$. All experiments were performed at $295^{\circ} \mathrm{K}$. The liquids are chosen to represent a range of molecular properties: isotropic, nonisotropic, polar, non-polar, symmetric and asymmetric. The sensitivity of the line shape of collision-induced scattering to these properties was examined. A summary of the molecular properties of the liquids is given in table 1 . 
Table 1. Summary of Molecular Properties

\begin{tabular}{|c|c|c|c|c|c|c|}
\hline & \multirow[t]{2}{*}{$\cdot$} & \multicolumn{2}{|c|}{ Potential Parameters } & \multicolumn{2}{|c|}{ Polarizabflityc } & \multirow{2}{*}{$\begin{array}{l}\text { Dipole } \\
\text { Moment } \\
\mu \\
\text { (Debye) }\end{array}$} \\
\hline & & $\begin{array}{c}\varepsilon \\
(0 . K)\end{array}$ & $\begin{array}{l}\sigma \\
(R)\end{array}$ & $\left(\mathrm{cm}^{3} \times 10^{25}\right)$ & $\left(\mathrm{cm}^{3} \times 10^{25}\right)$ & \\
\hline $\mathrm{CCl}_{4}$ & sph. top & $327^{a}$ & 5.88 & 105.0 & 0 & 0 \\
\hline $\mathrm{CHCl}_{3}$ & sym. top & $327^{a}$ & 5.43 & 82.3 & 23.3 & 1.1 \\
\hline $\mathrm{C}_{6} \mathrm{H}_{12}$ & sym. top & $324^{2}$ & 6.09 & 108.7 & - 24.3 & 0 \\
\hline $\mathrm{C}_{6} \mathrm{H}_{6}$ & sym. top & $440^{2}$ & 5.27 & 103.2 & 59.6 & 0 \\
\hline $\mathrm{C}_{7} \mathrm{H}_{8}$ & Asym.top & $.444^{b}$ & 5.79 & 122.6 & 73.6 & $0.4^{\circ}$ \\
\hline
\end{tabular}

aHirschfelder, J.0., Curtiss, C. F and Bird, R. B. 1954. Molecuiar Theory of Gases and Liquids (Wtiey, New York).

bestimated from data appearing in Reid, R. C. and Sherwood, T.K. 1966. The Properties of Gases and Liquids (MCGraw-Hill, New York).

CFabelinskii, I. L. 1968. Molecular Light Scattering (Plenum, New York).

dicclelian, A. L. 1963. Tables of Experimental Dipole Moments (W.H. Freeman). 
CHAPTER 4

METHOD OF ANALYSIS

Examples of depolarized spectra for the five liquids studied are shown in Figs. 4, 5, 6, 7 and 8 . These spectra are plotted with the estimated average noise subtracted and data points are shown every $2 \mathrm{~cm}^{-1}$. Fig. 9 contains spectra of all the liquids.

Fig. 10 is a plot of $I(\omega) / \omega^{q}$ versus frequency. for $\mathrm{CCl}_{4}$ (Tabisz et al. 1972). q can take on the values of $4 / 7$ and $12 / 7$ for electron overlap and fxame distortion respectively. The slope of the graph is given by $1 / \omega_{0}$. Bucaro and Litovitz obtained values of $\omega_{0}$ which agree with their theory by assuming a frame distortion origin for the induced polarizability, i.e. $q=12 / 7$. However, from Fig. 10, the $q=4 / 7$ (implying electron overlap) line obviously gives an improved relationship over the $q=12 / 7$ line because the quality of the fit is better and the linearity extends over a wider range. This result motivated the present analysis, a fitting procedure with all the parameters varying. This method is described in the following paragraphs.

\subsection{Method of Least Squares (Moore and Ziegler 1960; Bevington 1969)} Suppose that a function

$$
y=f\left(x_{1}, x_{2} \ldots x_{m} ; \alpha_{1}, \alpha_{2} \ldots \alpha_{p}\right)
$$

and a set of $n$ observations $\left(y_{i}, x_{1 i}, x_{2 i}, \ldots x_{m i}\right)$ are given where $i=1,2 \ldots n$ and $n>p$, the problem is to determine estimates of the $p$ parameters $\alpha_{k}$ such that the set of numbers will minimize the sum of the squares of the deviations of the observed $y_{i}$ from the 


\section{Figure 4}

Collision-induced scattering spectrum of $\mathrm{CCl}_{4}$ at $295^{\circ} \mathrm{K}$. Points are shown every $2 \mathrm{~cm}^{-1}$. 


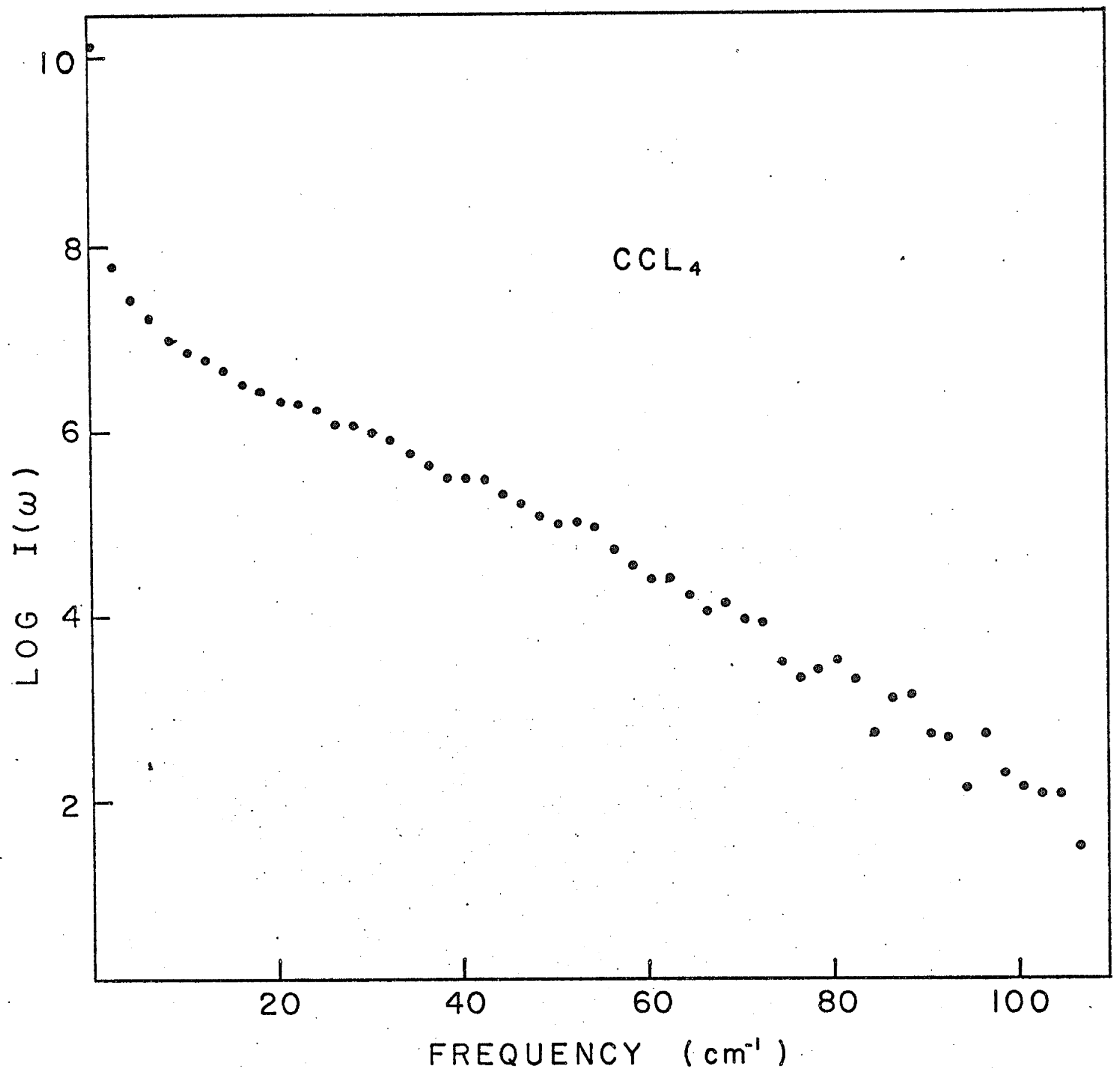

$\stackrel{\omega}{\circ}$ 
Figure 5

Collision-induced scattering spectrum of $\mathrm{C}_{7} \mathrm{H}_{8}$ at $295^{\circ} \mathrm{K}$. Points are shown every $2 \mathrm{~cm}^{-1}$. 


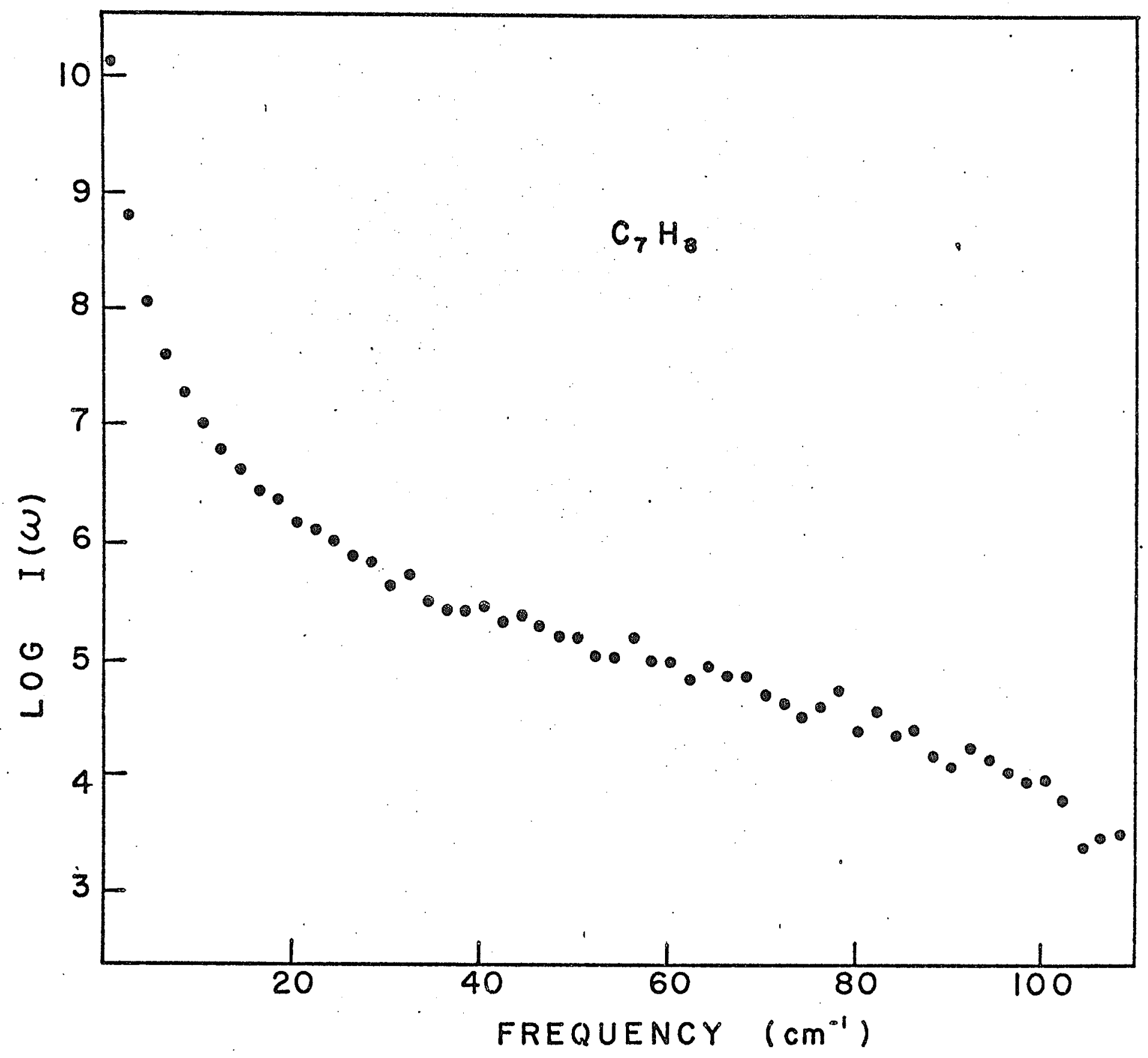


33.

Figure 6

Collision-induced scattering spectrum of $\mathrm{C}_{6} \mathrm{H}_{12}$ at $295^{\circ} \mathrm{K}$. Points are shown every $2 \mathrm{~cm}^{-1}$. 


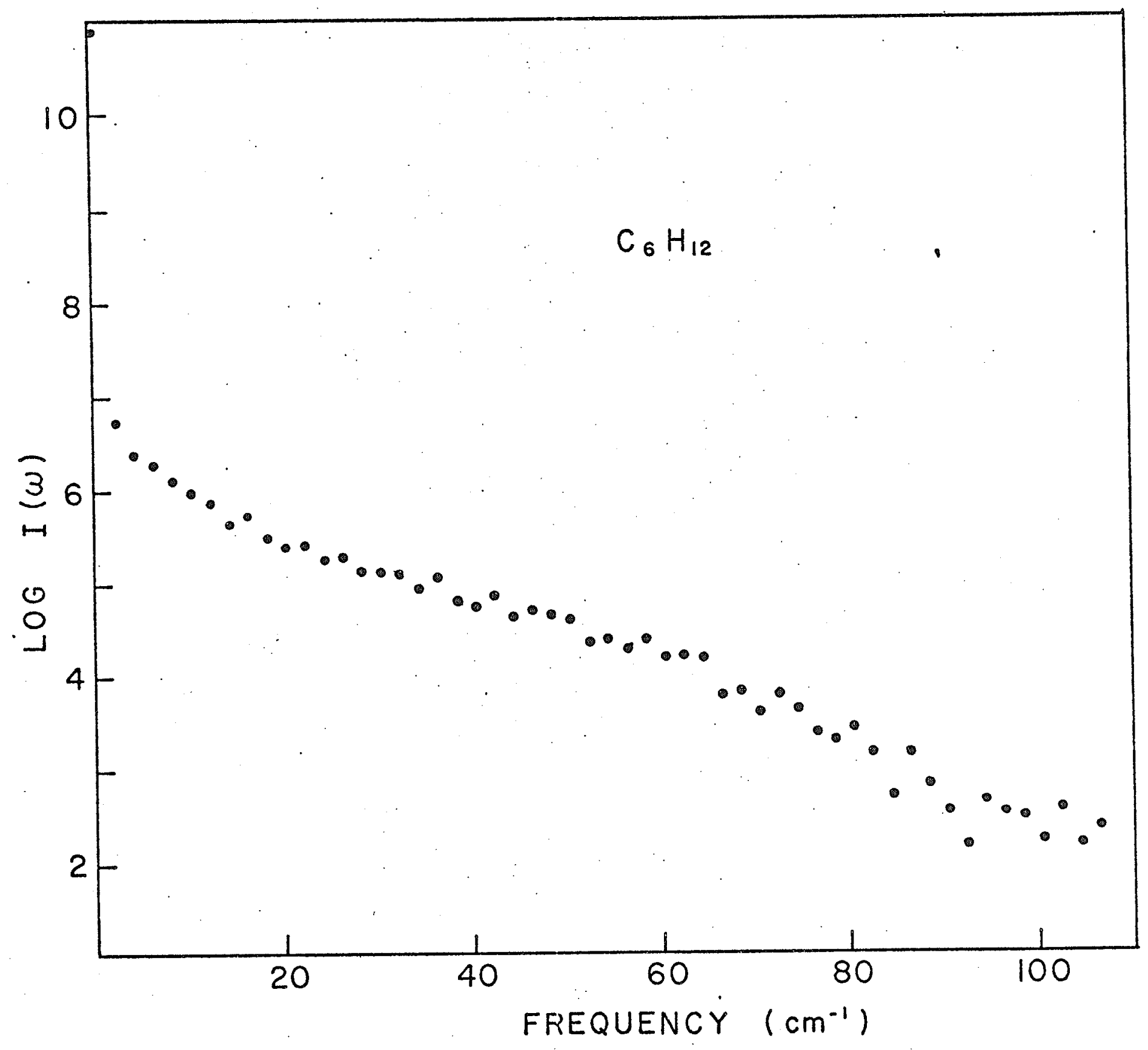


35.

Figure 7

Collision-induced scattering spectrum of $\mathrm{CHCl}_{3}$ at $295^{\circ} \mathrm{K}$. Points are shown every $2 \mathrm{~cm}^{-1}$. 


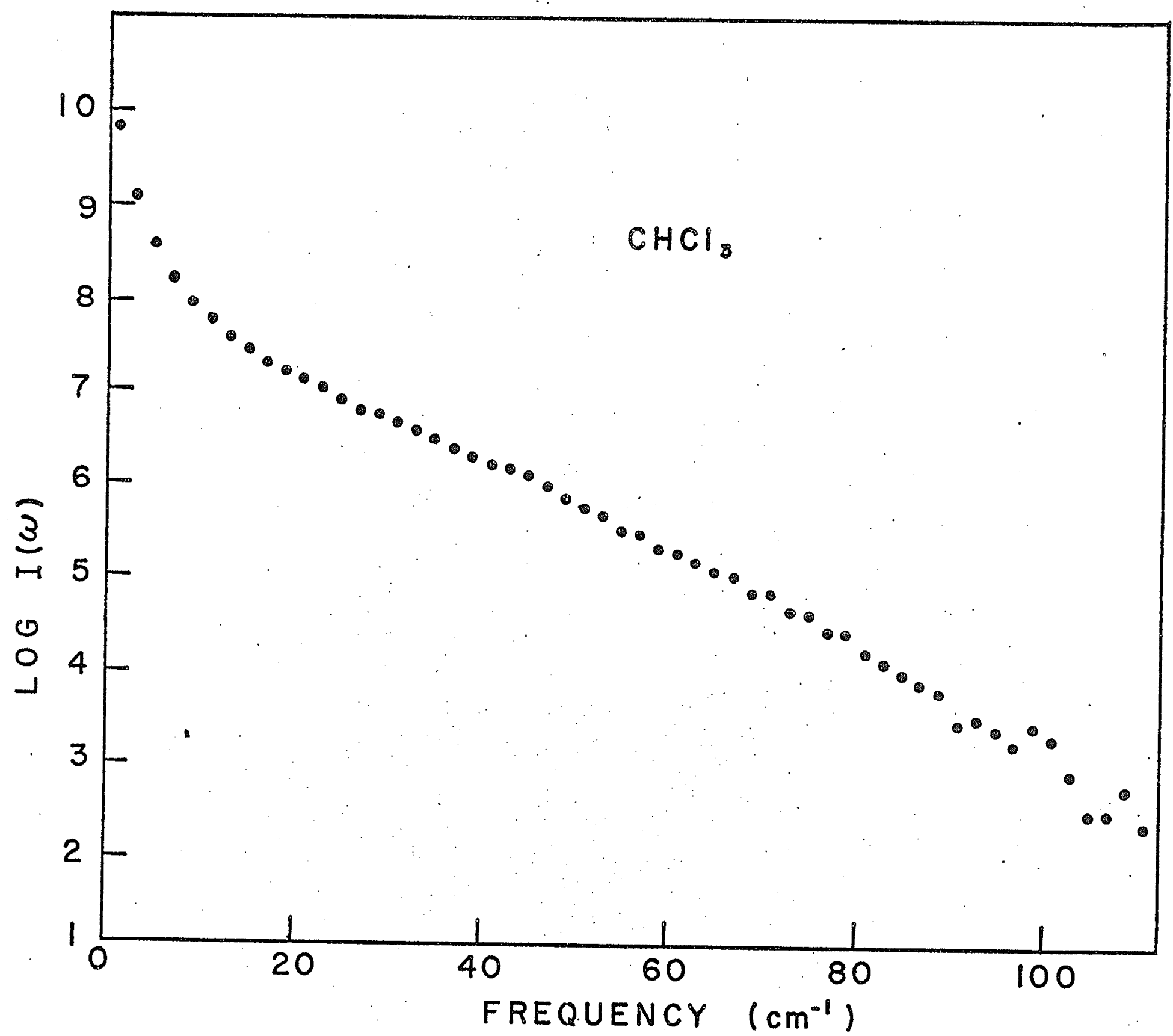


Figure 8

Collision-induced scattering spectrum of $\mathrm{C}_{6} \mathrm{H}_{6}$ at $295^{\circ} \mathrm{K}$. Points are shown every $2 \mathrm{~cm}^{-1}$. 


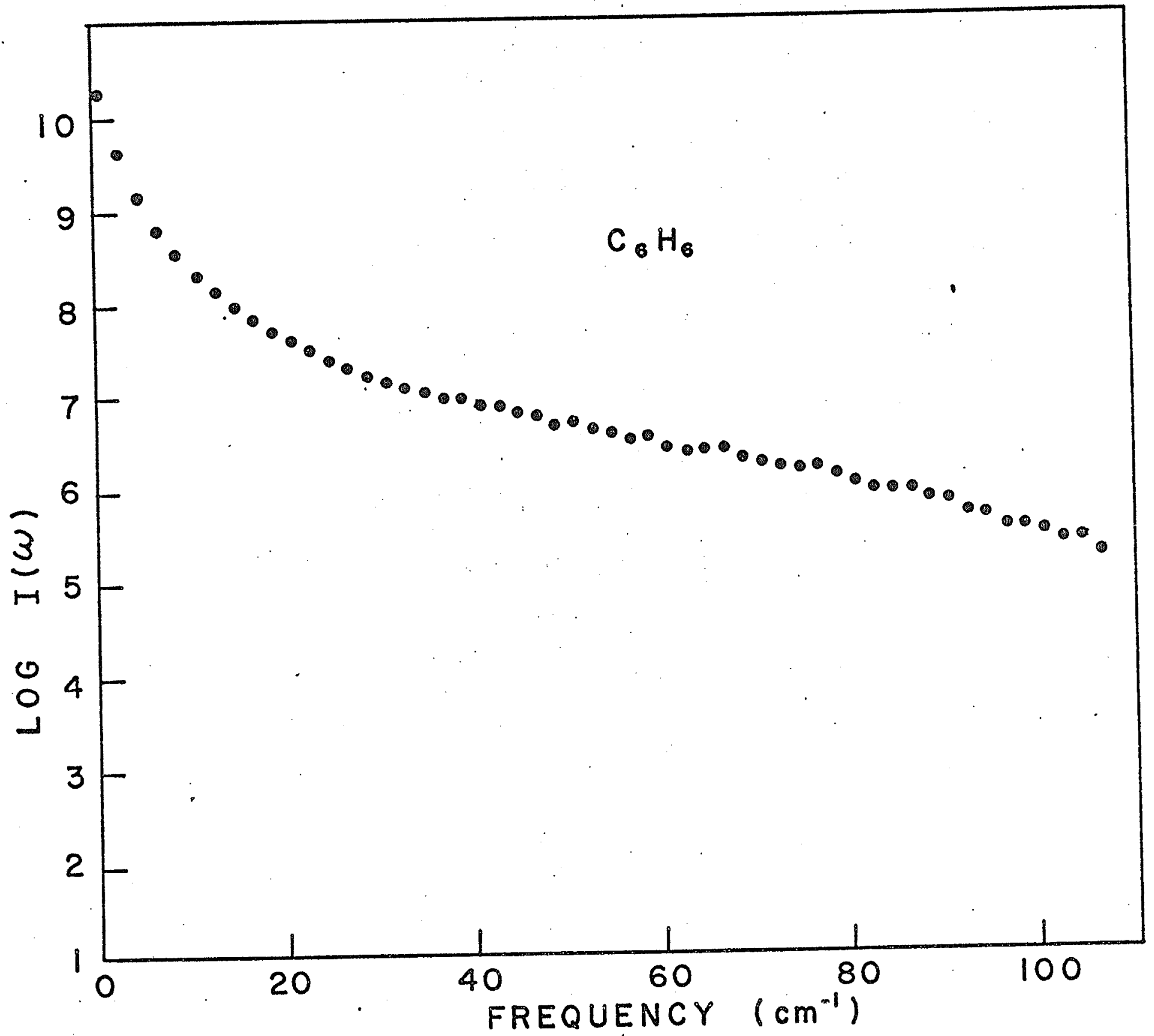




\section{Figure 9}

Typical observed spectra of the liquids at $295^{\circ} \mathrm{K}$. Points are shown every $1 \mathrm{~cm}^{-1}$. 


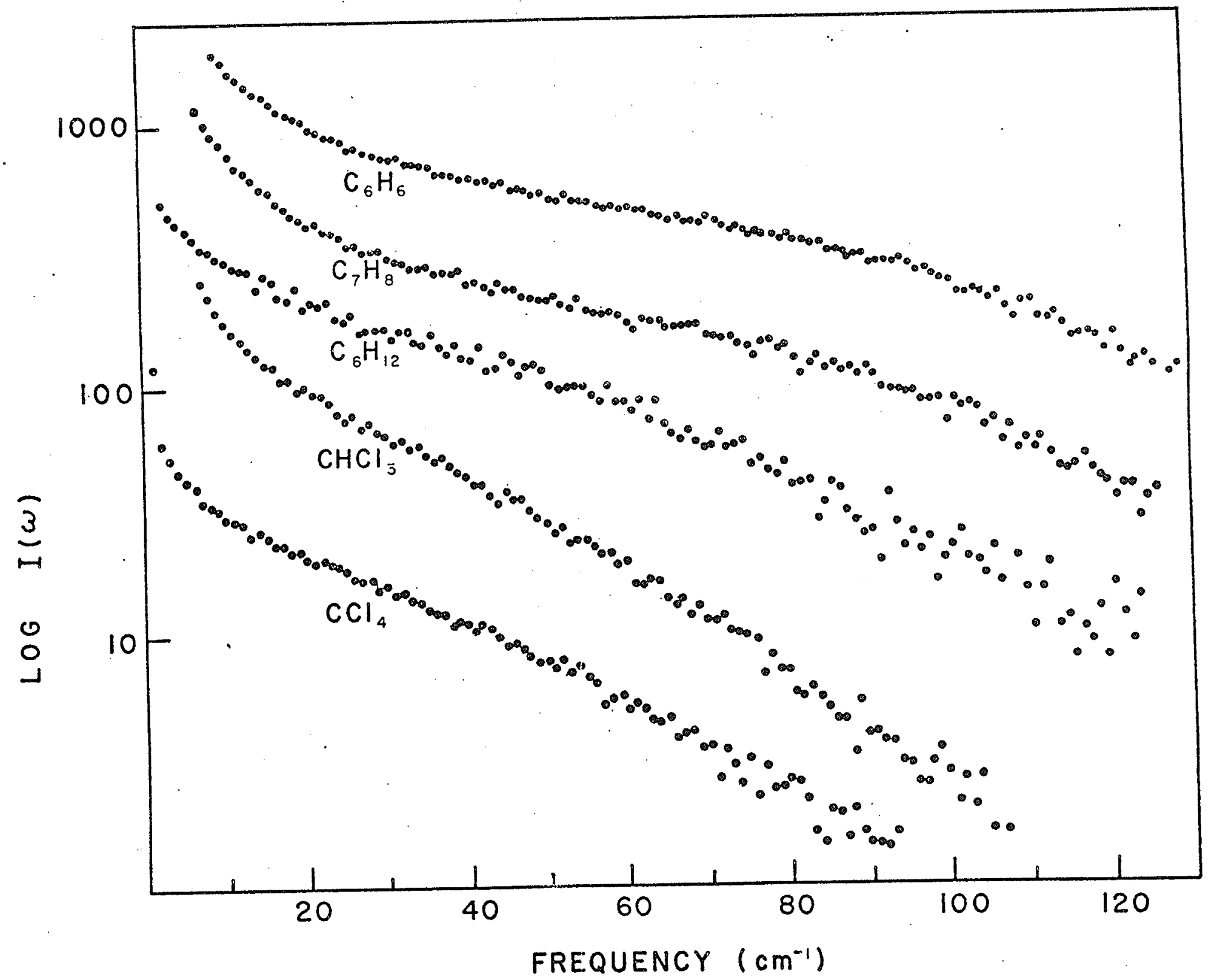




\section{Figure 10}

$\mathrm{I}(\omega) / \omega^{\mathrm{q}}$ for the observed spectrum of $\mathrm{CCl}_{4}$ at $293^{\circ} \mathrm{K}$ where $\mathrm{q}$ is $12 / 7$ and $4 / 7$ corresponding to $\Delta \alpha(r)$ varying as $r^{-13}$ and $\mathrm{r}^{-9}$ respectively. Data points are shown only every $4 \mathrm{~cm}^{-1}$. 
function. Mathematically one wishes to minimize

$$
Q=\sum_{i=1}^{n} w_{i}\left[y_{i}-f\left(x_{1 i} \ldots x_{m i} ; \alpha_{1} \ldots \alpha_{p}\right)\right]^{2}
$$

where $w_{i}$ are the weights associated with each of the $y_{i}$. The estimates of the $\alpha_{k}$ so obtained are denoted by $a_{k}$. The sum of the squares, $Q$, can be minimized by differentiating $Q$ with respect to each $\alpha_{k}$, setting the derivatives equal to zero, and solving the resulting set of $p$ simultaneous equations. Hence the set of equations,

$$
\frac{\partial Q}{\partial \alpha_{k}}=-2 \sum_{i=1}^{n} w_{i}\left(\frac{\partial Q}{\partial \alpha_{k}}\right)_{i}\left[y_{i}-f\left(x_{1 i}, \ldots x_{m i} ; \alpha_{1}, \ldots \alpha_{p}\right)\right]=0
$$

is obtained for $k=1,2, \ldots p$ and $\left(\frac{\partial f}{\partial \alpha_{k}}\right)$ denotes the $k$ th partial derivative for the $i$ th data point. Transposing and setting $f_{i}=f\left(x_{1 i}, \ldots, x_{m i}, \ldots \alpha_{1}, \ldots \alpha_{p}\right)$ the following normal equations are obtained,

$$
\begin{aligned}
& \sum_{i=1}^{n} w_{i} f_{i}\left(\frac{\partial f}{\partial \alpha_{1}}\right)_{i}=\sum_{i=1}^{n} w_{i} y_{i}\left(\frac{\partial f}{\partial \alpha_{1}}\right)_{i}, \\
& \sum_{i=1}^{n} w_{i} f_{i}\left(\frac{\partial f}{\partial \alpha_{2}}\right)_{i}=\sum_{i=1}^{n} w_{i} y_{i}\left(\frac{\partial f}{\partial \alpha_{2}}\right)_{i}, \\
& \sum_{i=1}^{n} w_{i} f_{i}\left(\frac{\partial f}{\partial \alpha_{p}}\right)_{i}=\sum_{i=1}^{n} w_{i} y_{i}\left(\frac{\partial f}{\partial \alpha_{p}}\right)_{i}
\end{aligned}
$$

When the parameters $\alpha_{k}$ appear as linear coefficients of the independent variables, the normal equations can be summarized by the matrix equation $A X=Y$ whose solution is $A=Y X^{-1}$. Howeyer, if the problem is non- 
linear, the test function must be linearized.

\subsection{The Gauss Method of Solving the Nonlinear Problem (Moore and}

Ziegler 1960)

Since the parameters to be varied to achieve the best fit appear non-1inearly in both the BL and Shin expressions, the Gauss method has been used to solve the resulting non-linear set of normal equations. The Gauss method consists essentially of linearizing the required test function with respect to each of the parameters by means of a truncated Taylor's series. Using initial estimates of the parameters to evaluate the coefficients of the expansion, new estimates are obtained. The process is repeated until some convergence criterion is satisfied.

Suppose, now, the problem is of the form in equation (4.1) and one wants to minimize $Q$ in (4.2). Some initial estimates of the parameters $a_{k, 0}$ are made, $a_{k, 0}$ being the $k$ th parameter. If (4.1) is expanded in a Taylor's series, the following equations are obtained:

$$
\begin{aligned}
\Delta y_{i, 0} & =y_{i}-f\left(x_{1 i}, x_{2 i}, \ldots x_{m i} ; a_{1,0}, a_{2, o}, \ldots a_{p, o}\right) \\
& =\left(\left.\frac{\partial f}{\partial \alpha_{1}}\right|_{i, 0}\right) \Delta a_{1,1}+\left(\left.\frac{\partial f}{\partial \alpha_{2}}\right|_{i, 0}\right) \Delta a_{2,1}+\ldots+\left(\left.\frac{\partial f}{\partial \alpha_{p}}\right|_{i, 0}\right) \Delta a_{p, 1} \\
& =\sum_{k=1}^{p}\left(\left.\frac{\partial f}{\partial \alpha_{k}}\right|_{i, 0}\right) \Delta a_{k, 1} \quad \text { for } i=1,2, \ldots n
\end{aligned}
$$

where $\left(\left.\frac{\partial f}{\partial \alpha_{k}}\right|_{i, 0}\right)$ is the partial derivative of (4.1) with respect to $\alpha_{k}$ and is evaluated at $\left(a_{1,0}, a_{2,0}, \ldots a_{p, o}\right)$. The non-linear problem has been reduced to a linear problem with $\Delta y_{i, 0}$ as the dependent variables, 
the $\left(\left.\frac{\partial f}{\partial \alpha_{k}}\right|_{i, 0}\right)$ as the independent variables, and the $\Delta a_{k, 1}$ are the parameters to be estimated. The normal equations are

$$
\begin{aligned}
& \Delta a_{1,1}\left[\sum_{i=1}^{n} w_{i}\left(\left.\frac{\partial f}{\partial \alpha_{1}}\right|_{i, 0}\right)^{2}\right]+\ldots+\Delta a_{p, 1}\left[\sum_{i=1}^{n} w_{i}\left(\left.\frac{\partial f}{\partial \alpha_{1}}\right|_{i, 0}\right)\left(\left.\frac{\partial f}{\partial \alpha_{p}}\right|_{i, 0}\right)\right] \\
& =\sum_{i=1}^{n} w_{i} \Delta y_{i, 0}\left(\left.\frac{\partial f}{\partial \alpha_{1}}\right|_{i, 0}\right) \\
& \Delta a_{1,1}\left[\sum_{i=1}^{n} w_{i}\left(\left.\frac{\partial f}{\partial \alpha_{p}}\right|_{i, 0}\right)\left(\left.\frac{\partial f}{\partial \alpha_{1}}\right|_{i, 0}\right)\right]+\ldots+\Delta a_{p, 1}\left[\sum_{i=1}^{n} w_{i}\left(\left.\frac{\partial f}{\partial \alpha_{p}}\right|_{i, 0}\right)^{2}\right] \\
& =\sum_{i=1}^{n} w_{i} \Delta y_{i, 0}\left(\left.\frac{\partial f}{\partial \alpha_{p}}\right|_{i, 0}\right)
\end{aligned}
$$

The above is a set of $p$ linear equations in $p$ unknowns and can be summarized in matrix notations as $B X=Z$ whose solution is $B=Z X^{-1}$. The elements of $B$ are $\Delta a_{1,1}, \Delta a_{2,1}, \ldots \Delta a_{p, 1}$ and those of $x$ are

$$
x_{j k}=x_{k j}=\sum_{i=1}^{n} w_{i}\left(\left.\frac{\partial f}{\partial \alpha_{j}}\right|_{i, 0}\right)\left(\left.\frac{\partial f}{\partial \alpha_{k}}\right|_{i, 0}\right)
$$

and those of $z$ are

$$
z_{k}=\sum_{i=1}^{n} w_{i} \Delta y_{i, 0}\left(\left.\frac{\partial f}{\partial \alpha_{k}}\right|_{i, 0}\right)
$$

A set of values $\Delta a_{k, 1}$ is obtained with which to modify each of the $a_{k, 0}$ i.e.

$$
a_{k, 1}=a_{k, 0}+h \Delta a_{k, 1}
$$


where $h$ is a constant. The process is repeated with the improved estimates of the $a_{k}$ until it has converged after $q$ iterations and the $\Delta a_{k, q}$ are all sufficiently small. $a_{k, q}$ is then taken to be the least squares estimate of $\alpha_{k}$.

\subsection{Details of the Computer Programne}

The attached computer program (Appendix 1) was used to analyze the data obtained from the experiment. The number of data points, $N$, the estimated dark noise, BACK, the dispersion, DSIG, and the position of the laser line in channel number, CHO, were first read into the computer. The intensity, Y(I), from each channel was then read in. The estimated dark noise was subtracted from the recorded signal. Then some initial estimates of the parameters were also entored with NPA specifying the number of parameters to be varied and IP(I) specifying which parameter is to be varied with the I running 1 to 5 . Following this a correction was wade for the intensity response of the monochromator-detector system. The intensity response of the system has been measured using a black body source of radiation; the correction was probably insignificant being less inan $3 \%$ over the range of $200 \mathrm{~cm}^{-1}$.

After the initialization of the program a rough estimate of the parameter $C(1)$, was made by finding the area under the curve of the intensity plotted against the frequency and then dividing this area by the calculated value of the intensity using all the initial estimate of the parameters. Then the $X$ and $Z$ matrices were formed after having calculated the derivatives of the intensity with respect to the various parameters. The inversion of the $X$ matrix was performed and 
the $B$ matrix was constructed. A control on the maximum change was applied to each element of the $B$ matrix until all the elements converged to a cortain desired value. Then the new parameters were computed. This was followed by the computation of the difference between the experimental and the computed intensity, the $\chi^{2}$ value, and the error in the parameters.

The experimental data was weighted by a factor $W$, equal to the reciprocal of the variance, which assuming Gaussian counting statistics is simply $1 / n$, where $n$ is the number of counts.

The quality of the fit was judged by calculating the estimated variance,

$$
S^{2}=\frac{1}{N-p} \sum_{i=1}^{N} w_{i}\left[y_{i}-f\left(a_{1}, a_{2} a_{3}\right)\right]^{2}
$$

where $f$ is the test function, $N$ is the number of data points and $p$ is the number of parameters varied. As the numerator has a $\chi^{2}$ distribution on $N-p$ degrees of freedom, $S^{2}$ should lie near unity. In order to test the computer program the intensity was calculated from the formula

$$
\mathrm{n}=A \omega^{B} \exp \left(-\frac{\omega}{\omega_{0}}\right)
$$

for each channel of the required range using known paraneters of $A$, $B$ and $\omega_{0}$. With the square root of the intensity, $\sqrt{n}$, as the input parameter; random numbers were then generated with the subroutine Gauss (from IBM scientific subroutine package) which gives a normally distributed random number which ranges from $-\sqrt{n}$ to $+\sqrt{n}$ and with a mean of zero and a cortain standard deviation. This random muber is then 
added onto the calculated intensity to simulate experimental data and the curve is fitted with the new intensity for the required range. It was found that the values of the parameters obtained from the fit agreed with the ones used to calculate the intensity. An example of the parameters obtained from the BL curve fitting is

$$
\begin{aligned}
& B=-0.26316 \pm 0.05863, \quad \text { and } \\
& \frac{I}{\omega_{0}}=0.04789 \pm 0.00095
\end{aligned}
$$

which agree with the ones used to calculate the intensity,

$$
\begin{aligned}
& B=-0.31060, \text { and } \\
& \frac{1}{\omega_{0}}=0.04762
\end{aligned}
$$

Similar results were obtained for the Shin fit when the same procedure is applied. 
CHAPTER 5

ANALYSIS AND DISCUSSION

In this chapter some details concerning the calculations of the Iine profile by Bucaro and Litovitz (BL) and by Shin are discussed and each compared with our observations.

\subsection{Bucaro and Litovitz Formulation for the Intensity of the Scattered $\underline{\text { Radiation }}$}

It was reported by Gornal1, Howard-Lock and Stoicheff (1970) and also by McTague, Fleury and DuPre (1969) that the intensity of the liquid spectrum decreases approximately exponentially with increasing frequency shift. Gersten (1971) pointed out the importance of the attractive forces which act between the colliding pair and showed that the intensity does not follow a simple exponential form. He then proposed a theory for the asymptotic line shape of the scattered light. However, because of the complexity of the expression which involves an integral of the modified Bessel function, others have made simplifying assumptions in order to compare theory with experiment.

Bucaro and Litovitz made an extensive study of collision-induced light scattering in some atomic and molecular liquids and concluded that the essential details of the phenomenon carry over from the gas to the liquid state. Taking a binary interaction picture with a Lennard-Jones potential and assuming zero impact parameter, they expressed the dipole moment induced during a collision. Then the energy spectrum of scattered light from such a dipole was obtained. The contribution from all the collisions follows upon multiplication 
by the collision ratc. Then assuming short-range interactions, i.c.

$$
\Delta \alpha(r) \propto r^{-m}
$$

where $m \gg 1$, Bucaro and Litovitz arrived at the following expression for the intensity of the scattered spectrum as a function of frequency,

$$
I(\omega) \propto \omega^{2}(\mathrm{~m}-7) / 7 \int_{0}^{\infty} \mathrm{d} v v \exp \left(-\left\{\frac{\mu v^{2}}{2 k^{\top} T}-\frac{\omega \pi \sigma}{6 v}\left[1-\frac{2}{\pi} \arctan \left(\frac{2 \varepsilon}{\mu v^{2}}\right)^{\frac{1}{2}}\right]\right\}\right)
$$

In the above expression $v$ is the relative velocity of the colliding atoms, $k$ the Boltzmann constant, $\mu$ the reduced mass, $T$ the temperature, and $\varepsilon$ and $\sigma$ are the standard force constants in the Lennard-Jones potential. This expression was then evaluated by a numerical integration for each of the liquids studied. In each case the integral at high frequencies was found to be of the following form

$$
I(\omega) \propto \omega^{2(m-7) / 7} \exp \left(-\frac{\omega}{\omega_{0}}\right)
$$

where

$$
\omega_{0}^{-1} \approx(1 / 6) \pi \sigma(\mu / \mathrm{kT})^{\frac{1}{2}}\left[1-(2 / \pi) \arctan \left(\frac{2 \varepsilon}{\mathrm{k} T}\right)^{\frac{1}{2}}\right\rfloor
$$

with $\omega_{0}$ different for different systems. The constant $\omega_{0}$ is inversely proportional to a characteristic time associated with the duration of the interaction giving rise to $\Delta \alpha$. The assumption of zero impact parameter is not unreasonable because large impact paraneters are unlikely in the close-packed structure of the liquid molecules.

Thibeau et al. (1968, 1970) have shown that the simple DID model camot account for the intensity of the argon spectrum at 
moderate gas densities although the same model is successful at lower densities. This is because the DID mechanism suffers from cancellation effects at moderate or high gas densities. They explained that, at low density, the field that a given atom experiences is produced by interaction with only one other atom. At high density, since the neighboring atoms are more spherically distributed, the field seen by a given atom becomes more symmetrical and a decrease in the depolarized lightscattering intensity occurs. Such a cancellation does not occur for the overlap type of interaction. Hence there may be a relative change of importance in the dipole-induced dipole and the electron overlap models. By assuming electron overlap effects in monatomic liquids and molecular frame distortions in molecular liquids, i.e. $m=9$ for argon and xenon and $m=I 3$ in the case of carbon tetrachloride, Bucaro and Litovitz obtained values of $\omega_{0}$ in excellent agreement with their calculations. Basically they have stated that collisioninduced scattered light exists in both monatomic and polyatomic liquids and that binary interaction remains important even in the liquid phase. Collision-induced light scattering is not restricted to isotropic atoms or molecules but is also present with anisotropic molecules as well.

In the present analysis of the experimental data, two fitting procedures were tried:

(i) $\omega_{0}$ and $m$ varied,

(ii) $m$ fixed at 13 and only $\omega_{0}$ varied.

Fits were made over several frequency intervals. The results are given in Table 2. The values shown represent averages over several spectra and the exrors quoted are the $99 \%$ confidence intervals. 
Table 2. Parameter obtained using the expression $I=A^{2(m-7) / 7} \exp \left(\omega / \omega_{0}\right)$

\begin{tabular}{|c|c|c|c|c|c|c|}
\hline . & $\begin{array}{c}\text { Frequency } \\
\text { Range } \\
\left(\mathrm{cm}^{-1}\right)\end{array}$ & $\mathrm{m}$ & $\begin{array}{c}\omega_{0} \\
\left(\mathrm{~cm}^{-1}\right)\end{array}$ & $s^{2}$ & $\begin{array}{l}m=13(\mathrm{fi} \\
\omega_{0} \\
\left(\mathrm{~cm}^{-1}\right)\end{array}$ & $s^{2}$ \\
\hline $\mathrm{CCl}_{4}$ & $\begin{array}{l}\text { calc. } \\
16 \rightarrow 100 \\
30 \rightarrow 80 \\
40 \rightarrow 100\end{array}$ & $\begin{array}{l}8.3 \pm 0.2 \\
9.6 \pm 0.5 \\
7.3 \pm 1.1\end{array}$ & $\begin{array}{l}9.2(10.3)^{*} \\
18.0 \pm 0.5 \\
15.2 \pm 0.8 \\
19.2 \pm 1.9\end{array}$ & $\begin{array}{l}2.16 \\
1.55 \\
1.50\end{array}$ & $\begin{array}{l}11.0 \pm 0.2 \\
11.9 \pm 0.1 \\
12.6 \pm 0.2\end{array}$ & $\begin{array}{r}28.1 \\
2.4 \\
2.3\end{array}$ \\
\hline $\mathrm{CHCl}_{3}$ & $\begin{array}{l}c^{\dagger} \\
40 \rightarrow 100\end{array}$ & $11.8 \pm 1.0$ & $\begin{array}{l}10.1(12.3)^{*} \\
13.7 \neq 0.9\end{array}$ & 0.83 & $12.7 \pm 0.2$ & 0.85 \\
\hline $\mathrm{C}_{6} \mathrm{H}_{12}$ & $\begin{array}{l}\text { calct } \\
16 \rightarrow 100 \\
30 \rightarrow 80 \\
40 \rightarrow 100 \\
40 \rightarrow 120\end{array}$ & $\begin{aligned} 8.0 & \pm 0.28 \\
9.5 & \pm 0.7 \\
13.0 & \pm 0.9 \\
12.9 & \pm 0.9\end{aligned}$ & $\begin{array}{l}10.6(13.3)^{*} \\
23.1 \pm 1.3 \\
20.3 \pm 2.1 \\
15.0 \pm 1.2 \\
15.3 \pm 0.9\end{array}$ & $\begin{array}{l}3.06 \\
1.17 \\
1.10 \\
1.08\end{array}$ & $\begin{array}{l}13.0 \pm 0.3 \\
14.4 \pm 0.2 \\
15.0 \pm 0.1 \\
15.1 \pm 0.1\end{array}$ & $\begin{array}{l}26.3 \\
2.3 \\
1.12 \\
1.14\end{array}$ \\
\hline $\mathrm{C}_{6} \mathrm{H}_{6}$ & $\begin{array}{l}\text { calc. } \\
40 \rightarrow 100 \\
40 \rightarrow 150\end{array}$ & $\begin{array}{r}9.3 \pm 0.96 \\
14.2 \pm 0.74\end{array}$ & $\begin{array}{l}14.4 \\
31.3 \pm 4.19 \\
18.5 \pm 0.98\end{array}$ & $\begin{array}{l}1.20 \\
2.69\end{array}$ & $\begin{array}{l}20.8 \pm 0.3 \\
20.1 \pm 0.2\end{array}$ & $\begin{array}{l}2.28 \\
2.93\end{array}$ \\
\hline $\mathrm{C}_{7} \mathrm{H}_{8}$ & $\begin{array}{l}\text { calc. } \\
40 \rightarrow 100 \\
50 \rightarrow 115\end{array}$ & $\begin{array}{l}10.1 \pm 1.7 \\
17.5 \pm 3.2\end{array}$ & $\begin{array}{l}12.1 \\
26.8 \pm 5.4 \\
14.8 \pm 2.5\end{array}$ & $\begin{array}{l}1.67 \\
1.09\end{array}$ & $\begin{array}{l}19.8 \pm 0.4 \\
19.9 \pm 0.6\end{array}$ & $\begin{array}{l}1.27 \\
1.26\end{array}$ \\
\hline
\end{tabular}

* Values in brackets are those calculated numerically, Bucaro and Litovitz (1971a)

+ Calculated using equation (5.3). 
There are two factors which limit the frequency range to which fits are appropriate. First, the expression (5.2) is derived under the assumption of short-range collisions, that is, of short collision times. Since short collision times would imply higher frequencies because $\omega \propto \frac{1}{\tau}$ where $\omega$ is the frequency and $\tau$ the collision time, this expression (5.2) is only applicable to the high frequency portion of the spectrum. Specifically, it is valid in the region where $\omega \gg \omega_{0}$. From Table 1 which summarizes the characteristics of the five liquids studied one can see that the molecules of four of them have a permanent anisotropy in the polarizability which gives rise to a depolarized component at low frequencies due to interrupted molecular libration and which is usually described by a Lorentzian or squared Lorentzian profile (see, for example, Simic-Glavaski and Jackson 1972). These aspects of the problem are illustrated by the results for the isotropic molecule $\mathrm{CCl}_{4}$ and the anisotropic $\mathrm{C}_{6} \mathrm{H}_{12}$. The test function does not provide a good description when the strirting point is taken at low frequencies $\left(16 \mathrm{~cm}^{-1}\right)$ with $S^{2}$ values of 2.16 for $\mathrm{CCl}_{4}$ and 3.06 for $\mathrm{C}_{6} \mathrm{H}_{12}$.

The values of the parameters giving the best fit do depend upon the frequency range. For the anisotropic molecules when the $m$ indicates a very short-range interaction, that is, $m \geq 12$, the values of $\omega_{0}$ are reasonably close to those predicted by (5.3). The $\mathrm{CHCl}_{3}$ and $\mathrm{C}_{6} \mathrm{H}_{12}$ results are in excellent agrecment with the observations of $\mathrm{BL}(1971)\left(13.1\right.$ and $14.8 \mathrm{~cm}^{-1}$, respectively). For $\mathrm{CCl}_{4}$, the parameters vary over the profile beyond $20 \mathrm{~cm}^{-1}$. If $\mathrm{m}$ is set cqual to 13, $w_{0}$ agrees with the observation of $13 \mathrm{~L},(1971), 11.9 \mathrm{~cm}^{-1}$, but the quality of the fit is considerably poorer with an $s^{2}$ value of 
2.4 compared to 1.5 .

These results are open to several possible interpretations. A plot of $1 / \omega_{0}$ versus

$$
\mu^{\frac{1}{2}} \sigma\left[1-(2 / \pi) \arctan \left(\frac{2 \varepsilon}{\mathrm{kT}}\right)^{\frac{1}{2}}\right]
$$

is shown in figure 11

According to equation (5.3), these quantities are proportional to one another. Indeed, the data do follow two distinct straight lines. The one corresponds to values of $1 / \omega_{0}$ obtained in fits where $m \geq 13$ and the other to instances where $m$ is found to be approximately 9 . For the anisotropic liquids as the fit includes more of the high frequency tail, the effective value of $m$ increases suggesting that shorter range interactions are responsible for the scattering. On the contrary, for $\mathrm{CCl}_{4}$, the high frequency tail is best fitted by a low $\mathrm{m}, 7.3$. In the range 30 to $80 \mathrm{~cm}^{-1}$, that is for the major portion of the $\mathrm{CCl}_{4}$ profile, the $\mathrm{m} \sim 9$ agrees with Gornal et al. (1968). The conclusion could be that there are several contributions to the scattering each arising from a different $r$-dependent term in the total expression for $\Delta \alpha$. However, in all cases the higher $m$ corresponds to a lower $\omega_{0}$ and, hence, a longer collision time; this is the inverse of the behaviour expected from simple collision dynamics. Hence, the interpretation is somewhat unsatisfactory.

\subsection{Shin Formulation of the Scattered Intensity}

Bucaro and Litovitz carried out the calculation numerically to compare the theory with experimental data. They did not explicitly derive equation (5.2). Starting from the expression for the intensity 
55.

Figure 11

A plot of $1 / \omega_{0}$ versus

$X=(\mu)^{1 / 2} \sigma\left[1-(2 / \pi) \arctan (2 \varepsilon / k T)^{1 / 2}\right]$

$\left(\mathrm{gm}^{1 / 2}-\mathrm{cm}\right)$

$\omega_{0}$ is obtained by fitting equation (5.2) to the experimental data. 


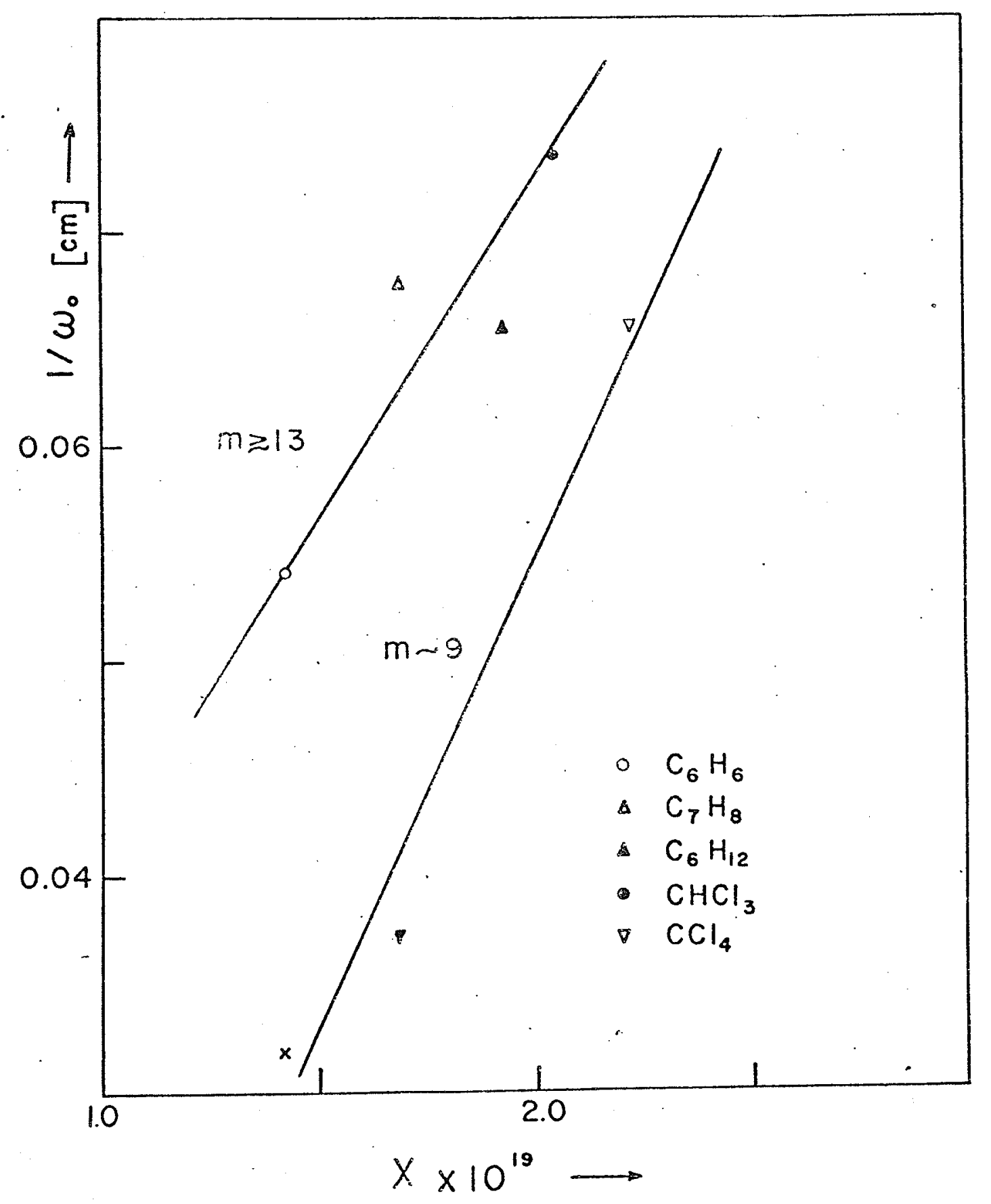


of the spectrum written as a statistical average, i.e.

$$
I(\omega, T)=C_{2} \int_{0}^{\infty} \int_{0}^{\infty} N(b, v) x\left|\int_{-\infty}^{\infty} \mu^{i n d}[r(t)] \exp (i \omega t) d t\right|^{2} d b d v
$$

and using the isolated binary collisions model of light scattering in liquids, Shin has developed a more exact and analytical expression for the intensity. In equation $(5,4) \mathrm{N}(\mathrm{b}, \mathrm{v})$ is the number of collisions per unit volume per unit time with impact parameter $b$ and relative velocity $v, \mu^{\text {ind }}$ is the induced dipole moment function and $C_{2}$ is the coefficient of the expression of the energy spectrum of scattered light. In order to evaluate the Fourier transform in equation (5.4), the trajectory $r(t)$ was determined using a short-range interaction and a Lennard-Jones potential. The Fourier transform was then found to be

$$
\int_{-\infty}^{\infty} \mu^{i n d}[r(t)] \exp (i \omega t) d t=\frac{2 \pi C}{\sigma^{m} \Gamma\left(\frac{1}{7} m\right)}\left[\frac{1}{14} \sigma\left(\frac{\mu}{2 D}\right)^{\frac{1}{2}}\right]^{m / 7} \omega^{(m-7) / 7} \exp (-\omega \tau)
$$

The thermal average of expression (5.5) is then taken and finally Shin, using the Laplace integration technique, arrived at the result

$$
\left.I \propto \omega^{(2(m-7) / 7)}+(4 / 19) \exp (-C \omega)^{12 / 19}+D \omega^{6 / 19}\right)
$$

where

$$
C=2.714 \frac{\beta}{\mathrm{kT}^{7 / 19}}
$$




$$
\begin{aligned}
& D=1.346 \frac{(\varepsilon \beta)^{1 / 2}}{\mathrm{kT}^{13 / 19}} \\
& \beta=\left[\frac{\Gamma(19 / 12)}{\Gamma(13 / 12)} \frac{(4 \varepsilon)^{1 / 12} \sigma}{12}(2 \pi \mu)^{1 / 2} \mathrm{k}\right]^{12 / 19}
\end{aligned}
$$

In the exponent of equation (5.6) the first term is the leading term and is due to the repulsive energy of the Lennard-Jones potential and the second term is due to the attractive energy. It should be pointed out that in both equations (5.2) and (5.4) the exponential term is independent of the form $\Delta \alpha$ 。

Equation (5.6) was fitted to our data by the Gauss method in the following ways:

(i) $\mathrm{m}$ fixed at 13 and $\mathrm{C}$ and $\mathrm{D}$ varied, (ii) $m$ varied and $C$ and $D$ fixed at the values given by $(5.7),(5.8)$ and $(5.9)$

The results are presented in table 3 .

As with the BL formulation, fits close to the laser frequency are poor and the parameters giving the best fit can vary across the profile of a given liquid. With $m$ set at 13 for three of the anisotropic molecules, the values of $C$ and $D$ are much greater than the predictions of (5.7) and (5.8). Therefore, if $r^{-13}$ is the correct form of $\Delta \alpha$, then the intermolecular potential or molecular dynamics used in the calculation do not accurately describe the physical situation. For $\mathrm{CCl}_{4}$, the Shin expression fits with essentially one set of parameters and the value of $\mathrm{C}$ obtained agrees with the predicted $\mathrm{C}$ value. For anisotropic molecules the observed $C$ values are somewhat higher than the predicted ones and also tend to increase with 
Table 3. Parameters obtained using the expression

$$
I=A \omega^{2(m-7) / 7+4 / 19} \exp \left(-C \omega^{12 / 19}+D \omega^{6 / 19}\right)
$$

\begin{tabular}{|c|c|c|c|c|c|c|c|}
\hline & $\begin{array}{c}\text { Frequency } \\
\text { Range } \\
\left(\mathrm{cm}^{-1}\right)\end{array}$ & $\underset{\left(\mathrm{cm}^{12} / 19\right)}{\mathrm{C}}$ & $\begin{array}{r}I \\
\left(\mathrm{~cm}^{6 / 1}\right.\end{array}$ & $\begin{array}{l}\text { D } \\
19)\end{array}$ & $s^{2}$ & $\mathrm{~m}$ & $\begin{array}{l}\text { fixed) } \\
s^{2}\end{array}$ \\
\hline $\mathrm{CCI}_{4}$ & $\begin{array}{l}\text { calc. * } \\
16 \rightarrow 100 \\
30 \rightarrow 80 \\
40 \rightarrow 100\end{array}$ & $\begin{array}{l}0.771 \\
0.50 \pm 0.03 \\
0.76 \pm 0.07 \\
0.76 \pm 0.07\end{array}$ & $\begin{array}{r}0.756 \\
-0.59 \\
1.15 \\
0.10\end{array}$ & $\begin{array}{l} \pm 0.2 \\
\pm 0.46 \\
\pm 1.22\end{array}$ & $\begin{array}{l}2.85 \\
1.56 \\
1.44\end{array}$ & & \\
\hline $\mathrm{CHCI}_{3}$ & $\underset{40 \rightarrow 100}{\operatorname{calc} c^{*}}$ & $\begin{array}{l}0.679 \\
1.07 \pm 0.37\end{array}$ & $\begin{array}{l}0.709 \\
3.44\end{array}$ & \pm 1.2 & 0.832 & $12.6 \pm 0.2$ & 1.01 \\
\hline $\mathrm{C}_{6} \mathrm{H}_{12}$ & $\begin{array}{l}\text { calc. * } \\
16 \rightarrow 100 \\
30 \rightarrow 80 \\
40 \rightarrow 100 \\
40 \rightarrow 120\end{array}$ & $\begin{array}{l}0.656 \\
0.366 \\
0.56 \pm 0.2 \\
1.04 \pm 0.16 \\
0.99 \pm 0.12\end{array}$ & $\begin{array}{c}0.693 \\
-1.24 \\
0.42 \\
3.61 \\
3.33\end{array}$ & $\begin{array}{l} \\
\pm 1.3 \\
\pm 1.16 \\
\pm 0.87\end{array}$ & $\begin{array}{l}6.67 \\
1.34 \\
1.07 \\
0.98\end{array}$ & $\begin{array}{l}14.6 \pm 0.4 \\
14.4 \pm 0.5 \\
14.3 \pm 0.5\end{array}$ & $\begin{array}{l}1.10 \\
1.02 \\
0.964\end{array}$ \\
\hline $\mathrm{C}_{6} \mathrm{H}_{6}$ & $\begin{array}{l}\text { calc. * } \\
40 \rightarrow 100 \\
40 \rightarrow 150\end{array}$ & $\begin{array}{l}0.591 \\
0.94 \pm 0.08\end{array}$ & $\begin{array}{l}0.765 \\
4.11\end{array}$ & \pm 0.6 & 3.13 & $\begin{array}{l}16.2 \pm 0.2 \\
15.7 \pm 0.2\end{array}$ & $\begin{array}{l}2.11 \\
4.67\end{array}$ \\
\hline $\mathrm{C}_{7} \mathrm{H}_{8}$ & $\begin{array}{l}\text { calc. * } \\
40 \rightarrow 100 \\
50 \rightarrow 115(\mathrm{~m}=17.5)\end{array}$ & $\begin{array}{l}0.661 \\
0.48 \pm 0.2 \\
0.991 \pm 0.6\end{array}$ & $\begin{array}{l}0.811 \\
0.65 \\
3.45\end{array}$ & $\begin{array}{l} \pm 1.3 \\
\pm 4.72\end{array}$ & 1.18 & $\begin{array}{l}17.6 \pm 0.3 \\
17.7 \pm 0.4\end{array}$ & $\begin{array}{l}1.34 \\
1.30\end{array}$ \\
\hline
\end{tabular}

* Calculated using equations [5.7], [5.8] and [5.9]. 
increasing high frequency tail. The $D$ values are higher than predicted and fluctuate. For $\mathrm{C}_{7} \mathrm{H}_{8}$ in the range $50 \rightarrow 115 \mathrm{~cm}^{-1}$ convergence cannot be achicved at $m=13$. However, with $m$ fixed at 17.5 the values obtained for $C$ and $D$ are $0.991 \pm 0.6$ and $3.45 \pm 4.72$ respectively. The errors in the values of $C$ and $D$ are relatively large.

The values found for $\mathrm{C}$ and $\mathrm{D}$ are plotted against $\left[\varepsilon^{1 / 19} \sigma^{12 / 19} \mu^{6 / 19}\right\rfloor$ and $\varepsilon^{1 / 2}\left[\varepsilon^{1 / 19} \sigma^{12 / 19} \mu^{6 / 19}\right]^{1 / 2}$ respectively in Fig. 12. Again reasonable straight line fits are obtained showing that the functional dependence of $C$ and $D$ on $\sigma, \varepsilon$ and $\mu$ is consistent with the model. For $\mathrm{CCl}_{4}$ uncertainty in $\mathrm{D}$ is large especially at high frequencies where it encompasses zero. In the exponential in the Shin expression the $-\mathrm{C} \omega^{12 / 19}$ term is due to repulsive forces while the $D \omega^{6 / 19}$ term describes the effects of longrange forces. Hence at higher frequencies, i.e. in the far wing of the tail the $D \omega^{6 / 19}$ term would be negligible compared with the $C \omega^{12 / 19}$ term. The statistics are not good enough to allow a precise determination of $D$.

When $C$ and $D$ are set at the values calculated from (5.7), (5.8) and (5.9), the $m$ giving the best fit stays constant over the high frequency profile and in the case of $\mathrm{C}_{6}{ }_{12}$ gives a better fit than by any othex expression. This situation is very appealing as (5.6) now holds beyond about $30 \mathrm{~cm}^{-1}$ with a single set of parameters. However, the $m$ required is near 13 for $\mathrm{CCl}_{4}$ and $\mathrm{CHCl}_{3}$, about 14.5 for $\mathrm{C}_{6} \mathrm{H}_{12}$ and even higher for $\mathrm{C}_{6} \mathrm{H}_{16}$. These values of $\mathrm{m}$ are close to those found with the BL function in the far tail but the r-dependence 


\section{Figure 12}

A plot of

(i) $\quad \mathrm{C}$ versus $\mathrm{Y}=\left[\varepsilon^{1 / 19} \sigma^{12 / 19} \mu^{6 / 19}\right]$

$$
\left(\mathrm{gm}^{7 / 19}-\mathrm{cm}^{14 / 19}-\sec ^{-2 / 19}\right) \text { and }
$$

(ii) $D$ versus $Z=\varepsilon^{1 / 2}\left[\varepsilon^{1 / 19} \sigma^{12 / 19} \mu^{6 / 19}\right]^{1 / 2}$

$$
\left(\mathrm{gm}^{13 / 19} \mathrm{~cm}^{26 / 19} \mathrm{sec}^{-20 / 19}\right)
$$

Only $\mathrm{C}$ is shown for $\mathrm{CCl}_{4}$ and $\mathrm{C}_{7} \mathrm{H}_{8}$

$C$ and $D$ are obtained by fitting equation (5.6)

to the experimental data. 


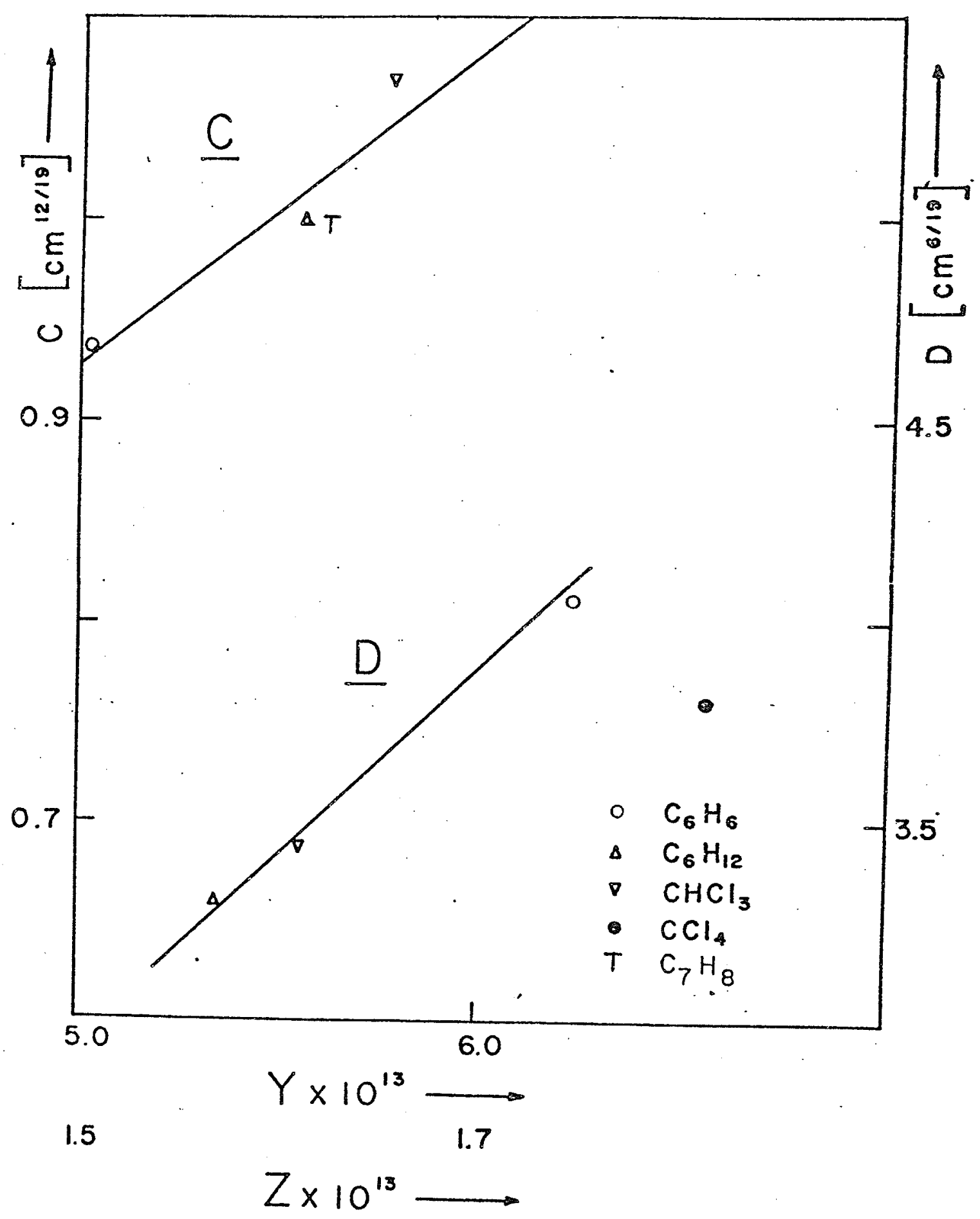


of $\Delta \alpha$ is now a strong function of the molecule.

That $C$ and $D$ are sensitive to temperature and local heating of the fluid by the laser beam might be a possible cause of error. However, they would be expected to decrease as $\mathrm{T}$ increases since

$$
\begin{aligned}
& C=2.714 \frac{\beta}{\mathrm{kT}^{7 / 19}}, \\
& D=1.346 \frac{(\varepsilon \beta)^{1 / 2}}{\mathrm{kT}^{13 / 19}},
\end{aligned}
$$

and

$$
\mathrm{dC}=-2.714\left(\frac{7}{19}\right) \frac{\beta}{\mathrm{k}} \frac{1}{\mathrm{~T}^{26 / 19}} \mathrm{dT},
$$

and

$$
\mathrm{dD}=-1.346\left(\frac{13}{19}\right) \frac{(\varepsilon \beta)^{1 / 2}}{\mathrm{k}} \frac{1}{\mathrm{~T}^{32 / 19}} \mathrm{dT}
$$

Hence the large values obtained with the anisotropic molecules cannot be attributed to this effect. 
CHAPTER 6

SUMMARY

Several schemes have been tried in order to reproduce the observed collision-induced scattering from five molecular liquids. A $x^{2}$ criterion has been used to assess the quality of the fits. In first applications of the isolated binary collision model an induced anisotropy having an $r^{-13}$ dependence could be used with a number of the molecular liquids. However, in the present work, to achieve the best fit using the BL equation, the induced anisotropy was found to range from $r^{-7}$ to $r^{-1.7}$ and varies not only between molecules but also over the high frequency profile of a given liquid. The Shin expression, being a more exact analytical formulation of the IBC model, should be more sensitive to the assumptions of the model. When applied with $m=13$, the coefficients of the terms within the exponent are higher than calculated. If the predicted coefficients are used, then the required $m$ varies between molecules but the major portion of the tail of the spectrum may be fitted with a single set of parameters. An interaction where $\Delta \alpha$ varies as $r^{-17}$ would be inconsistent since the $C$ and $D$ coefficients were obtained with a Lennard-Jones (6-12) potential. In fact, Gersten (1971) has clearly shown that the high frequency tail of collision-induced scattering due to the dipole-induced dipole mechanism in compressed rare gases should be quite sensitive to the nature of the short range part of the interaction potential. Thus, while the success of these fits is intriguing, their interpretation is ambiguous.

Hence different expression inplying different physical processes 
can adequately describe the observed spectra. The Shin expression is found to be successful with the isotropic molecule, $\mathrm{CCl}_{4}$, with the m consistent with the suggested value for frame distortion and it becomes less successful as the anisotropy increases. The BL expression is the better for the more anisotropic molecules $\mathrm{C}_{6} \mathrm{H}_{6}$ and $\mathrm{C}_{7} \mathrm{H}_{8}$. It should be pointed out that these latter two molecules are much more anisotropic than the others and hence, some contribution from the rather intense scattering due to the permanent anisotropy may extend into the far tail.

Finally, in the IBC model, the form of $\Delta \alpha$ and the molecular dynamics explicitly contribute to different factors in the expression for the intensity, $\omega^{x} \exp (\ldots)$. Nevertheless, as the above fits show, a change in the power of $\omega$ can significantly affect the terms within the exponential. Therefore, before a positive distinction between models can be made or before any reliable information on molecular dynamics in liquids can be extracted from the experimental results, a good theoretical estimate of the functional form of $\Delta \alpha$ for close collisions is necessary.

Very recently there has been suggestions from computer experiments in molecular dynamics that the isolated binary collision model is inadequate for liquid spectra. Berne, Bishop and Rahman (1973) evaluated the depolarized spectrum of scattered light and found that certain features of the spectrum can be associated with the dynamics of atomic motions in the fluid. Assuming that the argon atoms interact with a Lennard-Jones potential and with the $B(R)$ models suggested by Levine and Birnbaum (1971) and by O'Brien (1973), they calculated the correlation 
functions involving $\beta(R)$ molecular-dynamically and found that the major time dependence in the variation of the polarizability of the system comes from the correlated reorientation of pairs. Hence they conclude that the uncorrelated binary collision is inadequate and in dense systems the collision-induced polarizability may contain three, four and many atom contributions and cannot be ascribed entirely to two body interactions. They reaffirm that the collision-induced spectrum seems to be related to the density fluctuations in a fluid that destroy spherical symmetry around given atoms.

In another computer experiment the value of the polarizability anisotropy of a fluid was found by Alder, Weis and Strauss (1973) to be an increasingly smaller fraction of the dipole-induced dipole anisotropy as the density increases. They explained that at low density, since the field a given atom experiences is that produced by interaction with only one other atom, the light scattering per atom varies linearly with density. At high density, since the neighbouring atoms are more spherically distributed, the asymmetry is smaller and one expects a decrease in the light-scattering intensity. The light scattering per atom was then evaluated by calculating the dimensionless quantity

$$
S^{X Y}=\frac{\sigma^{6}}{N}<\left(\sum_{i \neq j}^{N} T_{i j}^{X Y}\right)^{2}>
$$

that is, the statistical average of the square of the value of one of the elements of the dipolar density fluctuation tensor for a system of $N$ spheres of diameter $\sigma$. The total value of $S$ was found to have a maximum value as expected at about the density which corresponds to 
the critical density of a real fluid. At higher densities, $S$ decreases due to the large cancellation between the pairwise, triplet and quadruplet terms. Hence they concluded that the distortion of the polarizability of an individual atom from spherical symmetry produces an anisotropy which opposes and dominates the dipolar density anisotropy in determining the intensity of the depolarized light scattering.

The present status of collision-induced scattering is reminiscent of the history of collision-induced absorption in highly symmetric molecules. The effect has been known for over twenty years (Welsh et al. 1949) but only after the interaction mechanism has been studied and elucidated under many conditions have accurate line shape calculations (Levine and Birnbaum 1967; Sears 1969) and applications to molecular dynamics been made (Mactaggart and Welsh 1973).

Further experimental studies of collision-induced scattering are required. It would seem, however, continued investigation of liquids would not be very profitable at this stage. The use of the isolated binary collision model and its associated simplifications, even if applicable, leads to ambiguous conclusions. The dual problems of the interaction mechanism and the molecular dynamics must be separated. The density change over a liquid temperature range is too small to result in significant variations in the observed spectra. In the gas phase, it is uncertain whether the eventual decrease in the depolarization ratio as the density increases is due to a change in the relative importance of long and short range interactions or due to the occurrence of multiple collisions. Careful studies of $I(\omega)$ and $\rho_{\ell}(\omega)$ at a low densities (50 amagats) with isotropic gases would be helpful. If the 
interaction mechanism can be clarified in the low density case where the dynamics are simple, then the results from more dense media would be more amenable to interpretation. A pressure cell is currently under construction in order to make such studies in this laboratory. 


\section{References}

Alder, B.J., Weis, J.J. and Strauss, H.L. 1973. Phys. Rev. A], 281. Berne, B.J., Bishop, M. and Rahman, A. 1973. J. Chem. Phys. 58, 2696.

Bevington, P.R. 1969. "Data Reduction and Error Analysis for the Physical Sciences" (McGraw-Hi11, New York).

Bucaro, J.A. and Litovitz, T.A. 1971a. J. Chem. Phys. 54, 3846. - $1971 \mathrm{~b}$ J. Chem. Phys. 55, 3585.

Buckingham, A.D. and Stephen, M.J. 1957. Trans.Faraday Soc. 53, 884.

Gabelnick, H.S. and Strauss, H.L. 1968. J. Chem.Phys. 49, 2334. Gelbart, W.M. 1972. J. Chem. Phys. 57, 699. Gersten, J.I. 1971. Phys. Rev。A4, 98.

Gornal1, W.S., Howard-Lock, H.F. and Stoicheff,B.P. 1970. Phys. Rev. AI, 1288.

Lallemand, P. 1970. Phys. Rev. Letters 25, 1079.

- 1971. J. Phys. (Paris) 32, 10.

Levine, H.B. and Birnbaum, G. 1967. Phys. Rev. 154, 72.

- 1968 Phys. Rev. Letters 20, 439.

- 1971 J. Chem. Phys. 55, 2914.

Mactaggart, J.W. and Welsh, H.L. 1973. Can. J. Phys. 51, 158.

McTague, J.P., Fleury, P.A. and DuPre, D.B. 1969. Phys. Rev. 188, 303.

McTague, J.P. and Birnbaum, G. 1971. Phys. Rev. A3, 1376. Moore, R.H. and Ziegler, R.K. 1960. LASL Report, LA-2367. O'Brien, E.F., Gutschick, V.P., McKoy, V. and McTague, J.P. 1973. 
"Polarizability of Interacting Atoms: Relation to CollisionInduced Light Scattering and Dielectric Models" (preprint). Pasmanter, R.A., Sampson, R. and Ben-Reuven, A. 1972. Chem. Phys. Letters 16,470 .

Sears, V.F. 1969. Can. J. Phys. 46, 2315.

Shin, H.K. 1972. J. Chem. Phys. 56, 2617.

- Simic-Giavaski, B. and Jackson, D.A. 1972. J. Phys. (Paris) 33.

Proceedings of the International Conference on Light Scattering by Fluids, Supplement to No. 2-3, C1-183.

Slusher, R.E., Surko, C.M. and Strautins, J. (unpublished). A preliminary account of this work has already been given by Gersten, J.I., Slusher, R.E. and Surko, C.M. 1970. Phys. Rev. Letters 25, 1739. Tabisz, G.C., Wall, W.R. and Shelton, D.P. 1972. Chem. Phys. Letters $15,387$.

Thibeau, M., Oksengorn, B. and Vodar, B. 1968. J. Phys. (Paris) 29, 287.

Thibeau, M., Tabisz, G.C., Oksengorn, B. and Vodar, B. 1970. J. Quant. Spectry. Radiative Transfer 10, 839 .

Vukcevich, M.R. 1971. Private communication quoted in Bucaro and Litovitz (1971a).

Welsh, H.L., Crawford, M.F. and Locke, J.L. 1949. Phys, Rev。 76, 580 . Wison, E.B., Jr., Decius, J.C. and Cross, P.C. 1955. "Molecular Vibrations" (McGraw-Hil1, New York).

Young, A.T. 1969. Applied Optics $\underline{8}, 2431$. 


\section{APPENDIX 1}

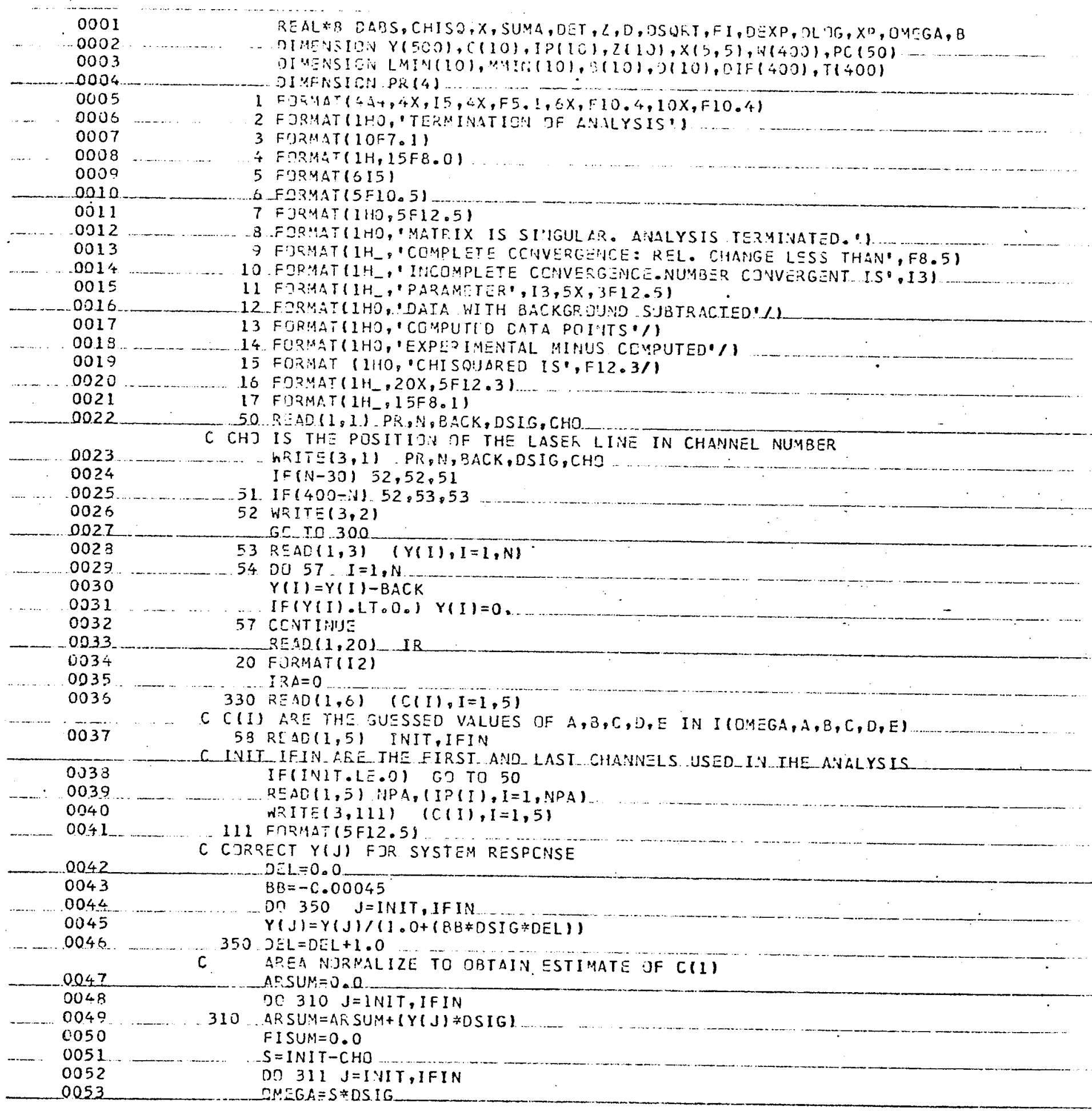




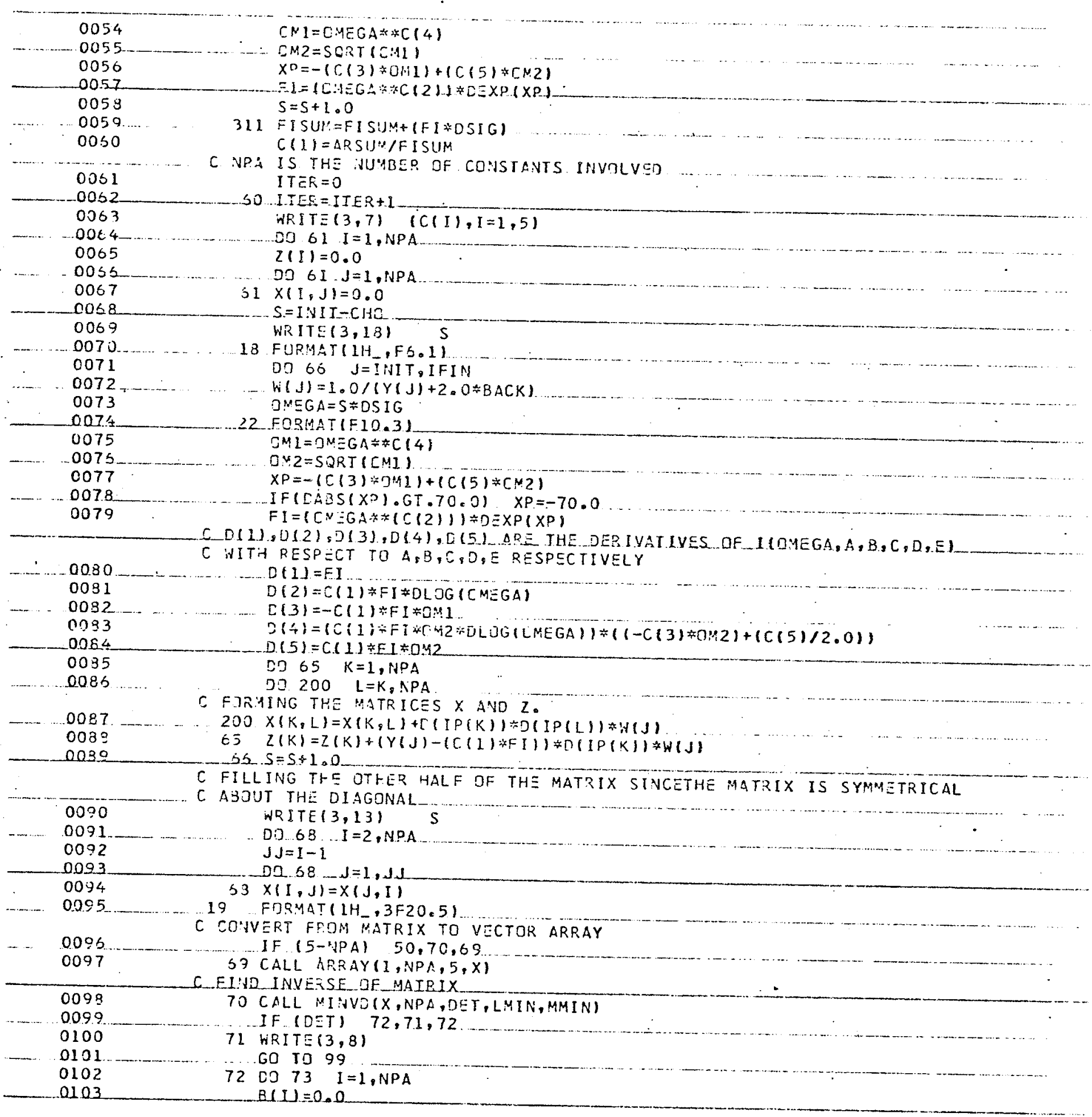




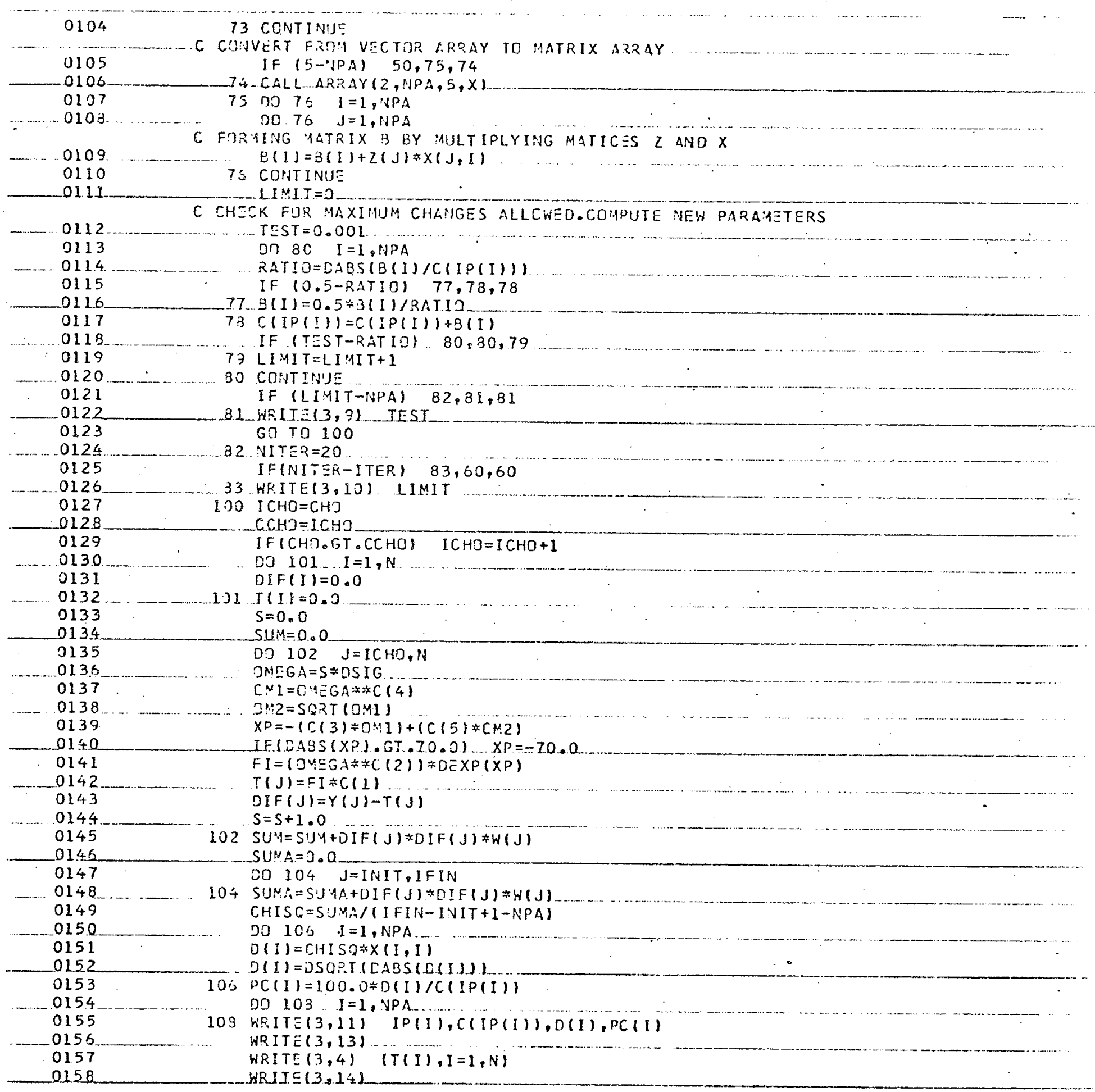




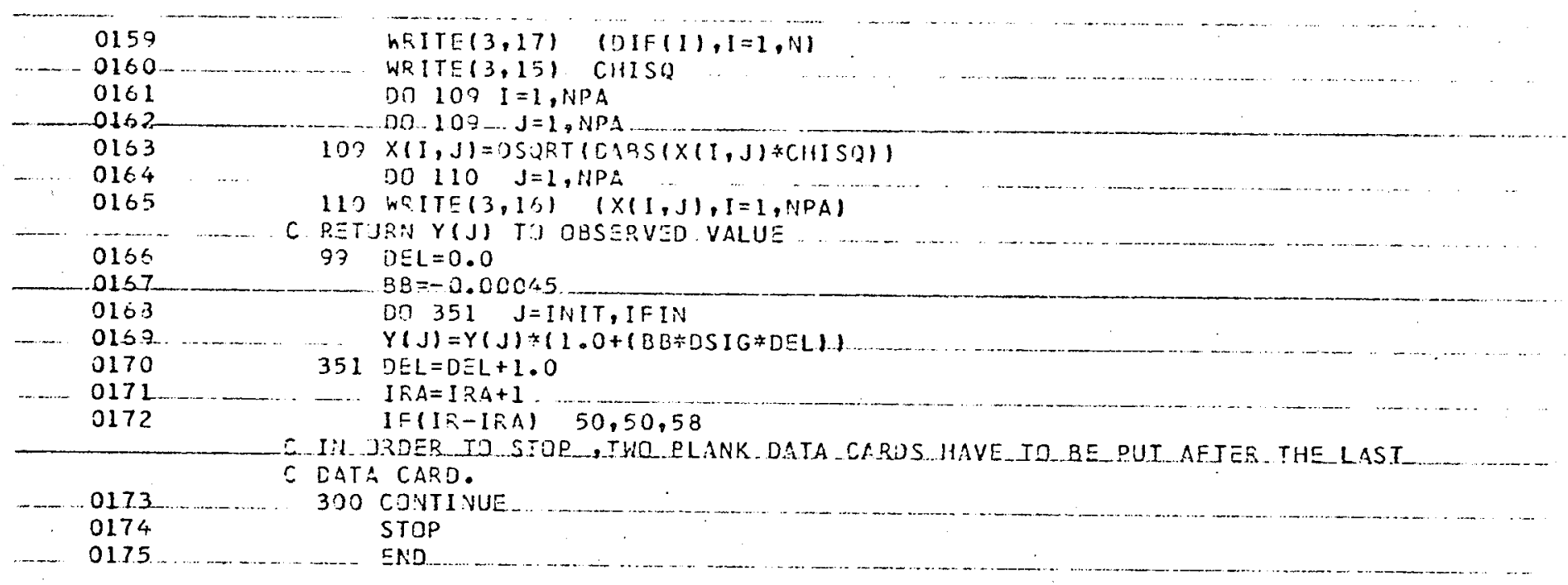




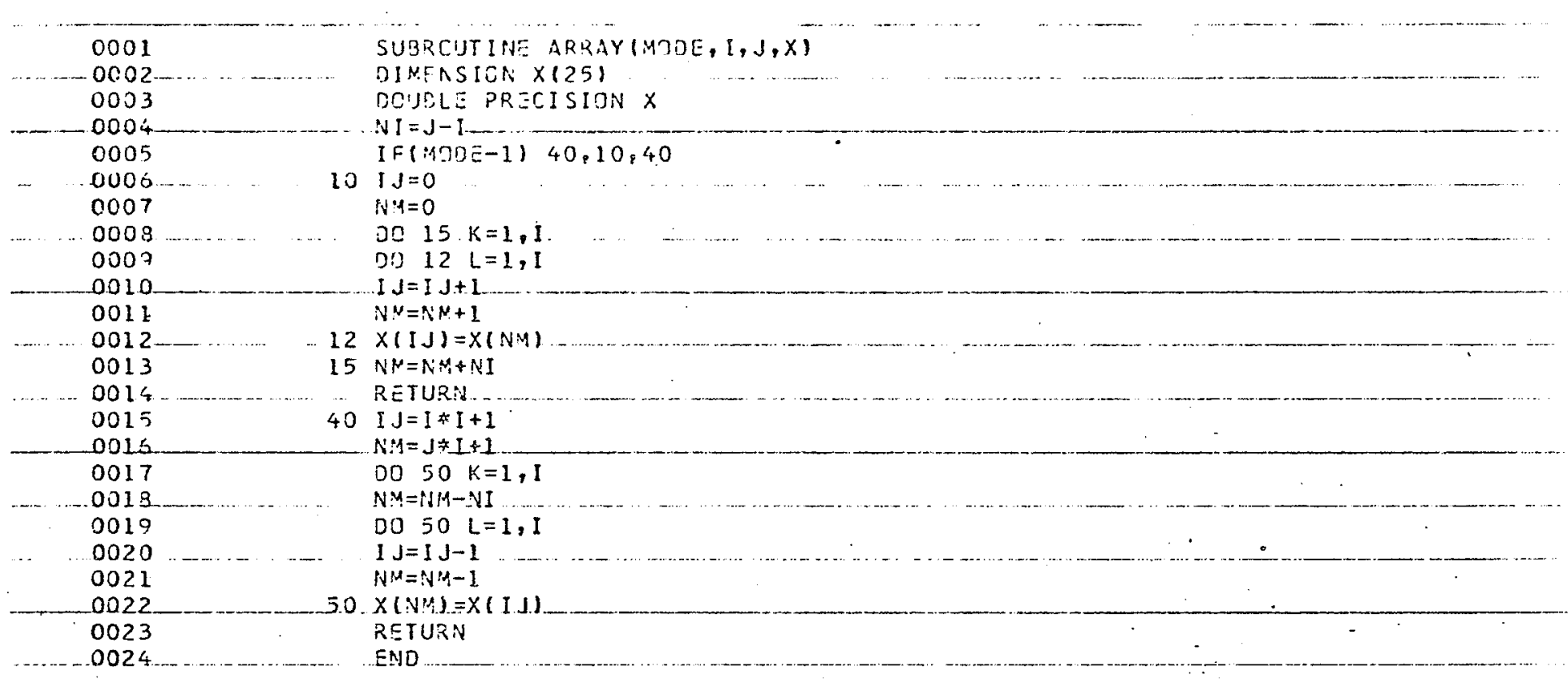
END 


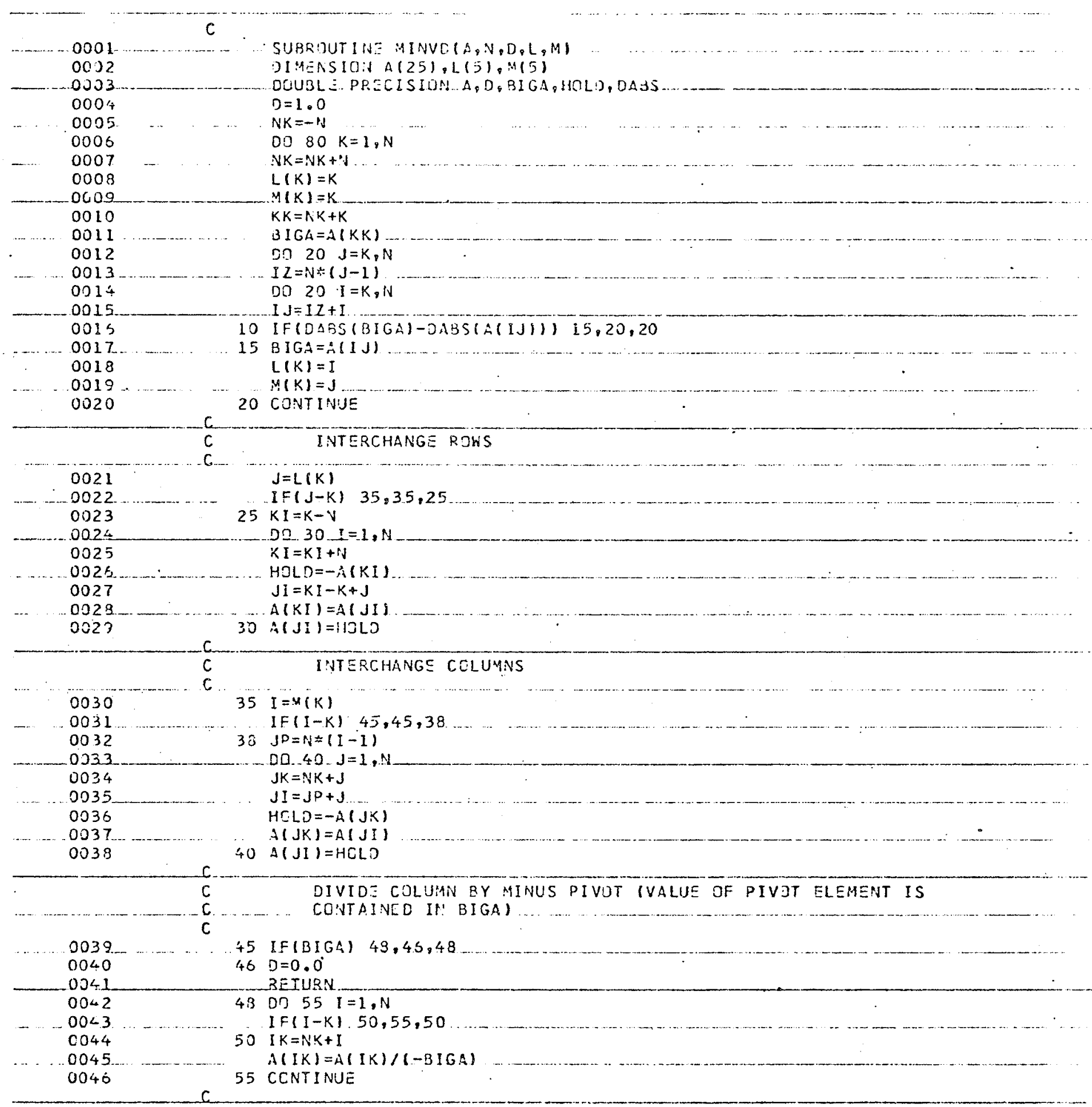




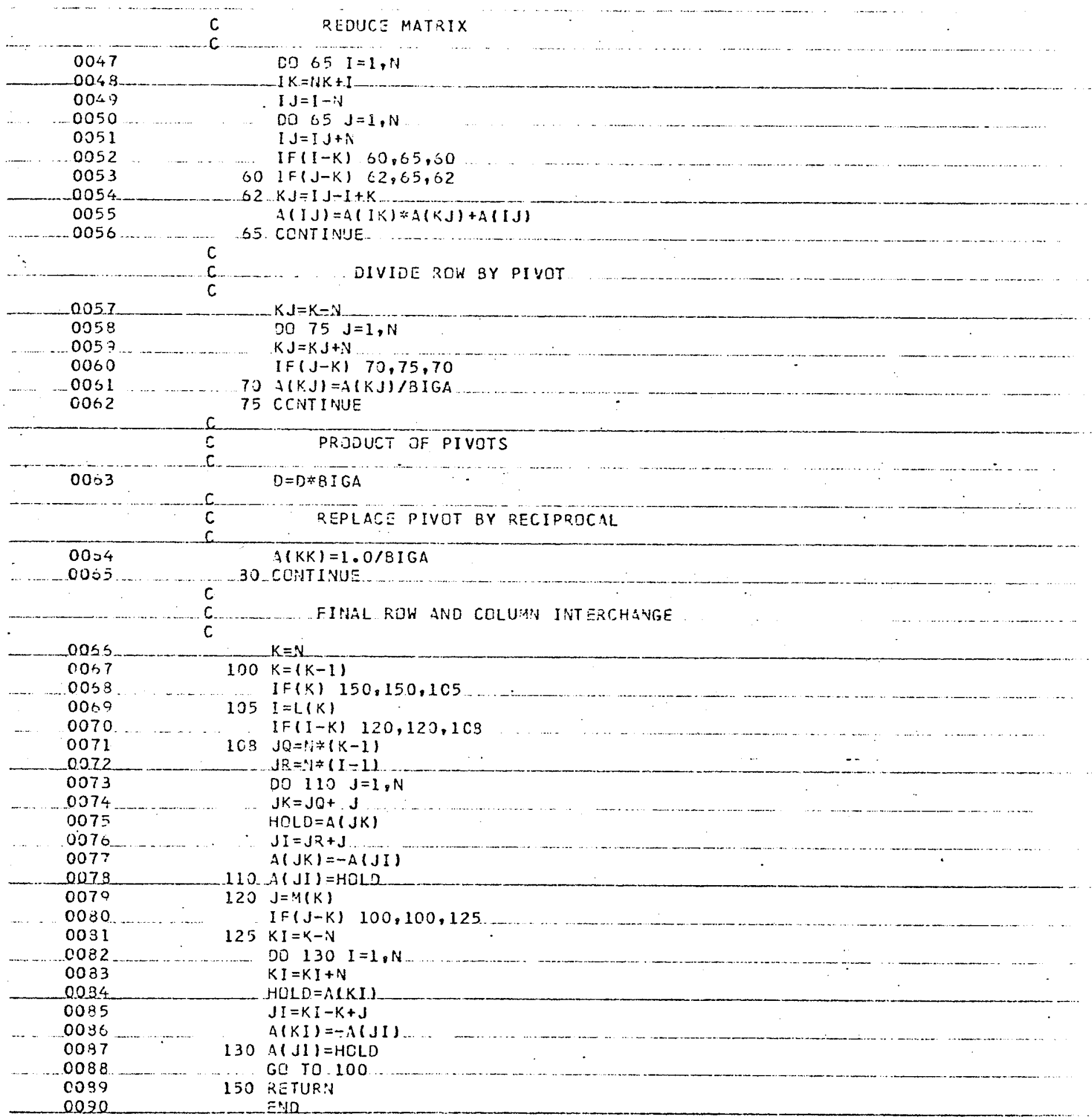


78.

APPENDIX 2

Reprinted from

ADVANCES IN RAMAN SPECTROSCOPY, VOLUME 1

(Proceedings of Third International Conference on Raman Spectroscopy,

Reims, France, September 1972)

Heyden \& Son Ltd., London 


\title{
60. THE SFECTRAL PROFILE OF COLISION. INDUCED WGHT SCATTERING FPON SOME MOLECULAR LIOUIDS
}

\author{
G. C. Tabisz, W. R. Wall, D. P. Shelton and J. Ho \\ Deparment of Piysics, Unilersity of Manitoba, \\ Wimipig, Manitoba, Canacla
}

\section{INTRODUCTION}

Collision-induced light seittering (CIS) is a newly identified phenomenon which has increasce the scope of the infomation obtainsble about derse media by seatteing methods. ${ }^{1-4}$ While fiec, optically isotopic atoms or molecules do not depolarize light, an anisotropy can be induced in the total polarimbility of a system of interacting atcms which produces a broad depolarized wing centred about the polarized Rayleigh line. Three

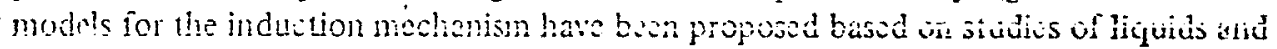
compressed gases.

(i) Dipole-induced dipole (DiD).,2.5 The anisotropy results from the interaction between electric dipoles indued in the atons by the incident light. The total field acting on a given atom is the sum of this extemal ligla field and the intemal field due to the induced cipoles in its neighbours. Orientational fluctuations in the net indued moment due to the atomic motion yield the depolarized scattering. This is a long-range interstion giving an effective piur polarizability which varies as $r^{-3}$ where $r$ is the distance between the atoms in a collision pair.

(ii) Electron Overlap (EO). ${ }^{3,4}$ The distortion of the elcctron clouds during close encounters is responsible for the inducel polarizability. Originally, the form $r^{2} \exp \left(-a r^{2}\right)$ was proposed but recently, an $r^{-9}$ dependence has been derived in the case of argon.

(iii) Frame Distortion. ${ }^{7}$ Bucaro and Litovit ${ }^{8,9}$ have made an extensive study or CJS in atomic and molecular liquids and have conclided that frame distontion during close collisions which gives a pair polarizability vary'ing is $r^{-13}$ is the principal induction mechanism in liquids composed of isotropic and non-isotropic molecules.

With the use of several simplifying assumpticns, pincipally binary collisions with zero impact parameter, Bucaro and litovitz were able to derive an expression for the intensity $I$ in the vings as a function of frequency $\omega$

$$
J(\omega) \propto \omega^{2 \mid(m-7) / 7]} \exp \left(-\omega / \omega_{0}\right)
$$

Where the induced incrementit polarizability is given by 
The Spectral Profile of Collision-Induced Light Scattering from Some Molecular Liquids

$$
\Delta a(r) \propto r^{-m}
$$

The constant $\omega_{0}$ is inversely proportional to a characteristic time associated with the duration of the interaction giving rise to $\Delta \alpha$.

In the analysis of their spectra, they assumed a value for $m$ and determined the corresponding $\omega_{0}$ from their data. As there is evidence from experiments on compressed gases $^{1,2}$ that the DID contribution diminishes at high densities, they assumed that, in the case of atomic liquids, the electron overlap effect predominates. They found that $m=9$ in the case of argon and xenon and $m=13$ in the case of molecular liquids gave values of $\omega_{0}$ in agreement with their calculations. This work is an excellent attempt to categorize CIS in liquids while providing insight into the interaction mechanisms responsible for the scattering. Primarily, it shows that binary interactions remain important even in the liquid phase.

Gersten ${ }^{10,11}$ has pointed out that in the spectra of compressed rare gases, different parts of the profile are affected by different details of the interaction.

Encouraged by the success of Bucaro and Litovitz but mindful that the complications envisaged by Gersten should apply to liquids as well as gases, we have repeated observations of the CIS spectra in pure $\mathrm{CCl}_{4}$ and $\mathrm{C}_{6} \mathrm{H}_{12}$ with the goal of further investigating the form of spectral profile.

\section{EXPERIMENTAL METHOD}

The experimental set-up is typical of laser Raman scattering studies. Light from an argon-ion laser (CRL No. 52) operating at about $3 / 4 \mathrm{~W}$ at $4880 \AA$ is passed through a halfwave plate and prism polarizer before being focused in the sample cell. The light scattered at $90^{\circ}$ is collected by an $f / 2$ system and brought to a double scanning monochromator (Jarrell-Ash No. 25-100) having a spectral slit width of $1 \mathrm{~cm}^{-1}$. The detector is a cooled EMI 6256B photomultiplier tube used in the photon counting mode. With the discriminator levels set for an optimum signal-to-noise ratio at low light intensities, the dark count is 1/3 c.p.s. The output is stored in a multichannel analyser (Victoreen No. ST 400M). whose channel advance is controlled by the stepping drive for the monochromator gratings. The geometry used is such that if the incident light is considered to travel in the $x$-direction, polarized in the $z$-direction, then observations are made in the $z$-direction. All experiments are performed at $295 \mathrm{~K}$.

\section{ANALYSIS OF RESULTS}

If a frame distortion origin for the induced polarizability is assumed, Eqn. (1) leads to a linear relationship between $\ln \left(I(\omega) / \omega^{12 n}\right)$ and $\omega$ with a slope $\left(-1 / \omega_{0}\right)$. When this test is applied to the data, it is found that $\omega_{0}=11.9 \mathrm{~cm}^{-1}$ for $\mathrm{CCl}_{4}$ and $15.0 \mathrm{~cm}^{-1}$ for $\mathrm{C}_{6} \mathrm{H}_{12}$ in excellent agreement with Bucaro and Litovitz (11.9 and $14.8 \mathrm{~cm}^{-1}$ respectively). The frequency range over which this linear relation holds is 30 to $80 \mathrm{~cm}^{-1}$ for $\mathrm{CCl}_{4}$ and 30 to $120 \mathrm{~cm}^{-1}$ for $\mathrm{C}_{6} \mathrm{H}_{12}$ (Fig. 1).

Frame distortion may not, however, be the only interaction mechanism contributing to the scattering. It was noted that much of the high frequency tail of the $\mathrm{CCl}_{4}$ could not be accounted for by the above method. When $m=9$ was tried, a value consistent with an electron overlap intcraction (at least for rare gas atoms), Eqn. (1) held over an increased frequericy range, $20 \mathrm{~cm}^{-1}$ to $100 \mathrm{~cm}^{-1}$. Moreover the quality of the fit was much improved 


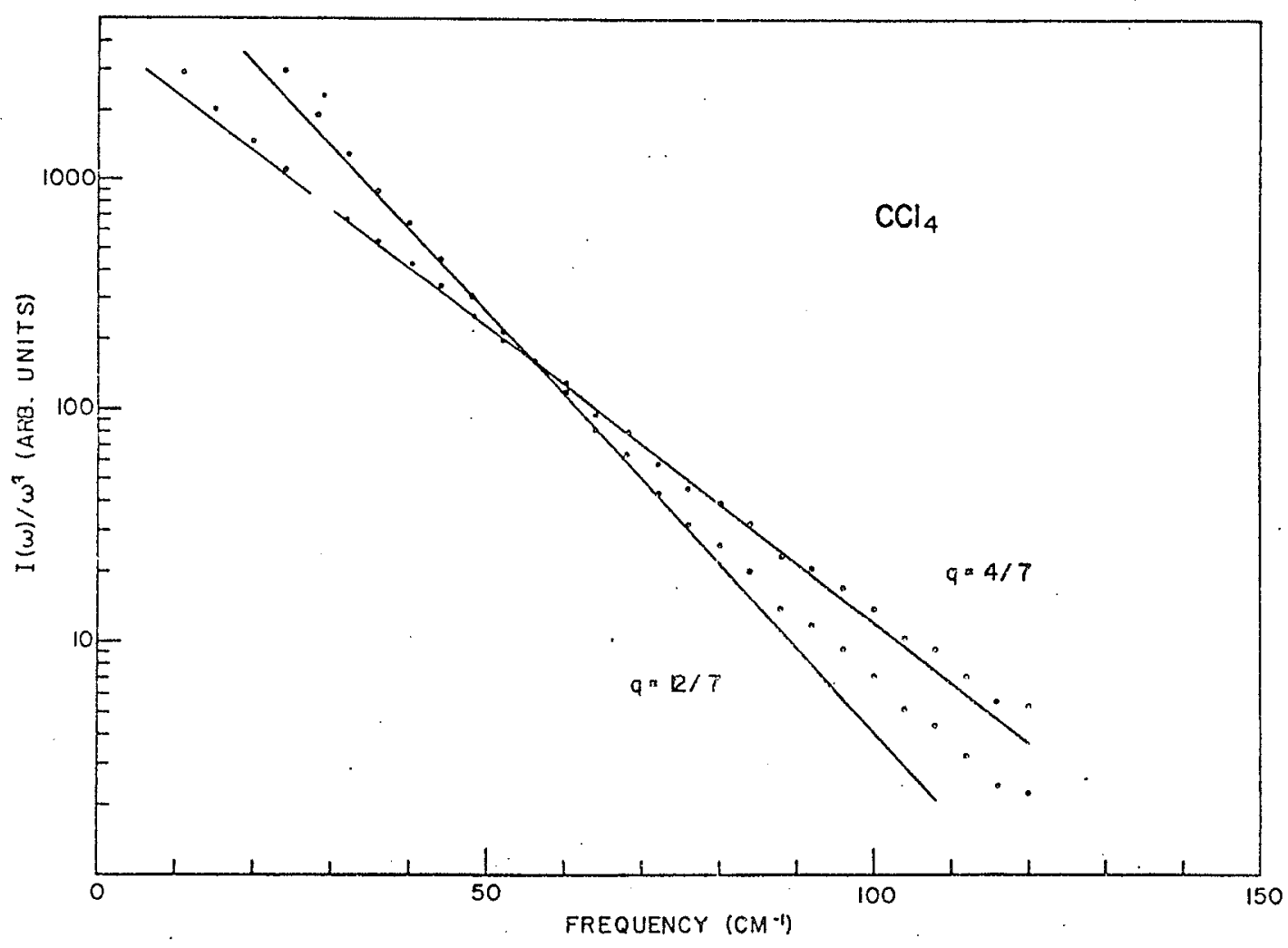

Fig. 1. $I(\omega) / \omega q$ for the observed spectrum of $\mathrm{CCl}_{4}$ at $295 \mathrm{~K}$ where $q$ is $12 / 7$ and $4 / 7$ corresponding to $\Delta \alpha(r)$ varying as $r^{-13}$ and $r^{-9}$ respectively. Data points are shown only every $4 \mathrm{~cm}^{-1}$. 
The Spectral Profile of Collision-Induced Light Scattering from Some Molecular Liquids

Table 1. Parameters obtained from the least squares fit with Eqn. (4).

\begin{tabular}{cccccc}
\hline & $\begin{array}{c}\text { Range } \\
\left(\mathrm{cm}^{-1}\right)\end{array}$ & \multicolumn{1}{c}{$q$} & $\begin{array}{c}\text { Effective } \\
m\end{array}$ & $\begin{array}{c}\omega_{0} \\
\left(\mathrm{~cm}^{-1}\right)\end{array}$ & $S^{2}$ \\
\hline & $16 \rightarrow 100$ & $0.36 \pm 0.05$ & $8.3 \pm 0.2$ & $18.0 \pm 0.46$ & 2.16 \\
$\mathrm{CCl}_{4}$ & $30 \rightarrow 80$ & $0.74 \pm 0.15$ & $9.6 \pm 0.5$ & $15.2 \pm 0.75$ & 1.55 \\
& $40 \rightarrow 100$ & $0.11 \pm 0.3$ & $7.3 \pm 1.1$ & $19.2 \pm 1.9$ & 1.50 \\
& $16 \rightarrow 100$ & $0.28 \pm 0.08$ & $7.9 \pm 0.28$ & $23.1 \pm 1.0$ & 3.06 \\
& $30 \rightarrow 80$ & $0.71 \pm 0.20$ & $9.4 \pm 0.68$ & $20.3 \pm 1.7$ & 1.17 \\
$\mathrm{C}_{6} \mathrm{H}_{12}$ & $40 \rightarrow 100$ & $1.73 \pm 0.27$ & $13.0 \pm 0.98$ & $15.0 \pm 0.97$ & 1.10 \\
& $40 \rightarrow 120$ & $1.68 \pm 0.24$ & $12.9 \pm 0.84$ & $15.2 \pm 0.91$ & 1.08 \\
\hline
\end{tabular}

over that with $m=13$. This result led us to perform the analysis based on the following considerations.

Most authors who have proposed mechanisms for collision-induced scattering conclude that the high frequency profile is described by an expression

$$
I(\omega)=f(\omega) \exp \left(-\omega / \omega_{0}\right)
$$

In the original model of Levine and Birnbaum, $f(\omega)$ is approximated by $\omega^{1 / 2}$; the more general function $\left.\omega^{2} \mid(m-7) / 7\right]^{2}$ shows clearly that the power of $\omega$ is a function of the induction process. Gersten ${ }^{11}$ has emphasized that the exponential factor in Eqn. (3) is model inciependent (alithougin the value of $\omega_{0}$ is not). The accurate determination of $f(\omega)$ should lead to meaningful information not available through the exponential. Because of the presence of this strong exponential factor, the exact form of $f(\omega)$ is difficult to extract from the experimental data.

In an effort to acquire some estimate of $f(\omega)$ directly from the observed spectra, we have assumed that the high frequency profile can be represented by

$$
I(\omega) \propto \omega^{q} \exp \left(-\omega / \omega_{0}\right)
$$

where both $q$ and $\omega_{0}$ were taken as variable parameters and made a least squares fit to the spectra. The results given in Table 1 are averages aver several spectra. The errors quoted represent a $99 \%$ confidence interval. For $\mathrm{C}_{6} \mathrm{H}_{12}$, the fits in the range $40 \mathrm{~cm}^{-1}$ to $120 \mathrm{~cm}^{-1}$ are the more significant as they are over a region sufficiently removed from the laser line to be free of contributions due to the permanent anisotropy of the molecule. The 'effective $m$ ' is determined by set ting

$$
q=(2 / 7)(m-7)
$$

$S^{2}$ is $\chi^{2}$ divided by the number of degrees of freedom.

\section{DISCUSSION}

The most striking result in Table 1 is the fact that for both liquids, the values of $q$ and $\omega_{0}$ depend on the frequency region over which the fit is made. For $\mathrm{C}_{6} \mathrm{H}_{12}$ the result in the range $4010120 \mathrm{~cm}^{-1}$ agrees with that of Bucaro and Litovitz. On the other hand, 
for $\mathrm{CCl}_{4}$, as more of the tail is included in the fit, the effective $m$ decreases and $\omega_{0}$ increases, nowhere approximating $m=13$ or $\omega_{0}=11.9 \mathrm{~cm}^{-1}$.

The failure of Eqn. (1) to hold over the entire tail with one set of values for $q$ and $\omega_{0}$ indicates that more than one type of interaction is operative in the seattering. For $\mathrm{C}_{6} \mathrm{H}_{12}$, short-range effects seem to be important with frame distortion especially dominant bcyond $40 \mathrm{~cm}^{-1}$. The theory of Bucaro and Litovitz holds well in this case: basically a single short-range interaction. For $\mathrm{CCl}_{4}$, both short- and long-range interactions contribute. Here the theory of Bucaro and Litovitz is not entirely applicable as their calculations of $\omega_{0}$ are valid only near the turning point. Judging from the effective $m$ values, the short-range part includes both electron overlap and frame distortion. The long-range interaction could be of the dipole-induced dipole type.

It should be recalled that because of symmetry effects, the intensity of the scattering through DID at liquid densitics is much less than that predicted from an extrapolation of the gas results using a quadratic dependence on density; however it should not necessarily vanish. In liquids the DID interaction would be effective through rapid local fluctuations in the positions of molecules, not describable by the trajectory dynamics used to derive Eqn. (1). Thus the spectral profile due to DID would broaden as the density increases and it would be expected to contribute to the far wings of spectrum.

The $\omega_{0}$ found for $40<\omega<100$ corresponds to a time of $2.8 \times 10^{-13} \mathrm{~s}$ which is of the order of magnitude of the time between collisions in a cell model for liquid $\mathrm{CCl}_{4}{ }^{12}$ the time between collisions would be the time characterizing these fluctuations in
positions.

Table 2. Parameters obtained from the least squares fit witli Eq̣n. (6).

\begin{tabular}{lllll}
\hline & \multicolumn{1}{c}{$\begin{array}{c}\text { Range } \\
\left(\mathrm{cm}^{-1}\right)\end{array}$} & \multicolumn{1}{c}{$B$} & \multicolumn{1}{c}{$C$} & $S^{2}$ \\
\hline $\mathrm{CCl}_{4}$ & $16 \rightarrow 100$ & $0.50 \pm 0.03$ & $-0.59 \pm 0.20$ & 2.85 \\
& $30 \rightarrow 80$ & $0.76 \pm 0.07$ & $1.15 \pm 0.46$ & 1.56 \\
& $40 \rightarrow 100$ & $0.76 \pm 0.07$ & $0.10 \pm 1.22$ & 1.44 \\
$\mathrm{C}_{6} \mathrm{H}_{12}$ & $16 \rightarrow 100$ & 0.37 & -1.24 & \\
& $30 \rightarrow 80$ & $0.56 \pm 0.20$ & $0.42 \pm 1.3$ & 6.67 \\
& $40 \rightarrow 100$ & $1.04 \pm 0.16$ & $3.61 \pm 1.2$ & 1.34 \\
& $40 \rightarrow 120$ & $0.99 \pm 0.12$ & $3.33 \pm 0.87$ & 0.98 \\
\hline
\end{tabular}

\section{Further analysis}

The expression developed by Shin ${ }^{13}$

$$
I(\omega) \propto \omega^{A} \exp \left(-B^{3} \omega^{12 / 19}+C \omega^{6 / 19}\right)
$$

was also fitted to the data with $A$ fixed for $m=13$. The results are given in Table 2 .

For $\mathrm{CCl}_{4}$ beyond $30 \mathrm{~cm}^{-1}$, the $B$ is in excellent agreement with predictions $(0.77)$ and while $C$ is uncertain, the expected value $(0.76)$ is bracketed. With $C_{0} H_{12}, B$ and $C$ are much larger than the model surgests $(0.66$ and 0.69$)$. The quality of the fit for both liquids is comparable to that using liqn. (4).

This result leads to the conclusion that frame distortion is important for $\mathrm{CCl}_{4}$ in the far tail. Since Eqn. $(6)$ is a more exact formulation within the binary collision model 
than is I:an. (1), it is mene sensitive to the assumptions mat. The neglect of the angular

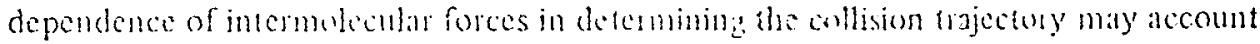
for the diserepincy in the case of the non-spierical motecule $\mathrm{C}_{6} \mathrm{H}_{12}$.

While both the Bucare and Liovitz and the Shin expessions can mathematically

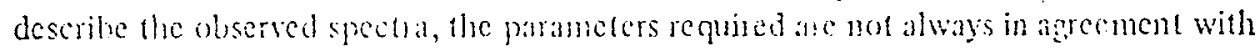
calculations and conntieting conclusions concrning the inderaction anise. The question of the line shape in colision-induced scatlering is still a problem.

\section{REFERENCES}

1 M. Thibean, B. Gksungon and B. Vodar, J. Phys. (Paris) 29, 287 (1968).

2 M. Thibeau, G. C. Tabisz, B. Okscngom and B. Vodu, J. Qntam. Spectry. Radiative Trarsscr 10, 839 (1970).

3 H. B. Lovine and G. Bimbaum, Phys Rev. Lotters 20, 439 (1968).

4 J.P. McTague and G. Bimbaum, Phy. Rev. Letters 21,661 (1968).

5 P. Lallemand, Ply's Rel. Lettcis 25, 1079 (1970); J. Phys. (Paris) 32, 10 (1971).

6 M. R. Vukccrich, private communicition quoted in jef. 8.

7 H. S. Gabehick and H. L. Strauss, J. Chem Plyss 49, 2334 (1968).

8 J. A. Buciro and T. A. Litovitz, J. Chem. Phys 54,38:6 (1971).

9 J. A. Bucaro and T. A. Litovitz, J. Chem. Phy's 55, 3585 (1971).

10 1. I. Gersten, R. E. Slusher and C. M. Surko, Phys. Re'setters 25, 1739 (1970).

11 J. I. Gerstein, Jlyys Rer, A4, 98 (1971).

12 F. J. Bartoli and T. A. Litovitz, J. Chem. Phys 56, 413 (1972).

. 13 II. K. Shiii, J. Chem. Phys. 56, 2617 (1972). 